\title{
Synthesis and insecticidal activity of diacylhydrazine derivatives containing a 3-bromo-1-(3-chloropyridin-2-yl)-1H-pyrazole scaffold
}

\author{
Yanyan Wang ${ }^{\dagger}$, Fangzhou Xu ${ }^{\dagger}$, Gang Yu, Jun Shi, Chuanhui Li, A’li Dai, Zhiqian Liu, Jiahong Xu, Fenghua Wang
} and Jian $\mathrm{Wu}^{*}$ (D)

\begin{abstract}
Background: The diacylhydrazine derivatives have attracted considerable attention in recently years due to their simple structure, low toxicity, and high insecticidal selectivity. As well as 3-bromo-1-(3-chloropyridin-2-yl)-1H-pyrazole is an important scaffold in many insecticidal molecules. In an effort to discover new molecules with good insecticidal activity, a series of diacylhydrazine derivatives containing a 3-bromo-1-(3-chloropyridin-2-yl)-1H-pyrazole scaffold was synthesized and bio-assayed.

Results: Bioassays demonstrated that some of the title compounds exhibited favorable insecticidal activities against Helicoverpa armigera and Plutella xylostella. The insecticidal activity of compounds $\mathbf{1 0 g}, \mathbf{1 0 h}$, and $\mathbf{1 0 w}$ against $\mathrm{H}$. armigera were $70.8,87.5$, and $79.2 \%$, respectively. Compounds $\mathbf{1 0 c}, \mathbf{1 0 e}, \mathbf{1 0 g}, \mathbf{1 0 h}, \mathbf{1 0 i}, \mathbf{1 0}$ jand $\mathbf{1 0 w}$ showed good larvicidal activity against $P$. xylostella. In particular, the $L C_{50}$ values of compounds $\mathbf{1 0 g}$, $\mathbf{1 0 h}$, and $\mathbf{1 0 w}$ were $27.49,23.67$, and $28.90 \mathrm{mg} \mathrm{L}^{-1}$, respectively.

Conclusions: A series of diacylhydrazine derivatives containing a 3-bromo-1-(3-chloropyridin-2-yl)-1H-pyrazole scaffold was synthesized and bio-assayed. The results of insecticidal tests revealed that the synthesized diacylhydrazine derivatives possessed weak to good insecticidal activities against H. armigera and P.xylostella. Compounds $\mathbf{1 0 g}$, $\mathbf{1 0 h}$, and 10x showed much higher insecticidal activity than tebufenozide, and exhibited considerable prospects for further optimization. Primary structure-activity relationship revealed that phenyl, 4-fluoro phenyl and four fluorophenyl showed positive influence on their insecticidal activities, and introduction of a heterocyclic ring (pyridine and pyrazole) showed negative impacts on their insecticidal effects.
\end{abstract}

Keywords: Diacylhydrazine, 3-Bromo-1-(3-chloropyridin-2-yl)-1H-pyrazole, Synthesis and insecticidal activity

\section{Background}

Diacylhydrazines are important of nonsteroidal ecdysone agonists inducing agent against lepidopteron, which show excellent insecticidal activity by inducing precocious molting. The earliest insecticidal diacylhydrazine

\footnotetext{
*Correspondence: wujian2691@126.com; jwu6@gzu.edu.cn ${ }^{\dagger}$ Yanyan Wang and Fangzhou Xu are co-first author for this manuscript Key Laboratory of Green Pesticide and Agricultural Bioengineering, Ministry of Education, Research and Development Center for Fine Chemicals, Guizhou University, Guiyang 550025, China
}

was developed by Rohm and Haas Company and named $\mathrm{RH}-5849$, which was also investigated for their mode of action $[1,2]$. Tebufenozide, the first commercialized diacylhydrazine as a specific insecticide for lepidopteron, was applied widely in many countries [3]. And then, several diacylhydrazine insecticides such as halofenozide, methoxyfenozide, chromafenozide, and JS-118 (Fig. 1), were also commercialized gradually [4-7]. Recently, diacylhydrazine derivatives have attracted considerable attention due to their simple structure, low toxicity, and 
<smiles>CC(C)(C)N(NC(=O)c1ccccc1)C(=O)c1ccccc1</smiles>

RH-5849<smiles>COc1cccc(C(=O)NN(C(=O)c2cc(C)cc(C)c2)C(C)(C)C)c1C</smiles>

methoxyfenozide<smiles>CNC(=O)c1cc(Cl)cc(C)c1NC(=O)c1cc(Br)nn1-c1ncccc1Cl</smiles>

chlorantraniliprole<smiles>CCc1ccc(C(=O)NN(C(=O)c2cc(C)cc(C)c2)C(C)(C)C)cc1</smiles>

tebufenozide<smiles>Cc1cc(C)cc(C(=O)N(NC(=O)c2ccc3c(c2C)CCCO3)C(C)(C)C)c1</smiles>

chromafenozide<smiles>CNC(=O)c1cc(C#N)cc(C)c1NC(=O)c1cc(Br)nn1-c1ncccc1Cl</smiles>

cyantraniliprole<smiles>CC(C)(C)N(NC(=O)c1ccc(Cl)cc1)C(=O)c1ccccc1</smiles>

halofenozide<smiles>Cc1cc(C)cc(C(=O)N(NC(=O)c2ccc3c(c2C)OC(C)C3)C(C)(C)C)c1</smiles>

JS-118<smiles>CNC(=O)c1cc(Cl)cc(Cl)c1NC(=O)c1cc(Br)nn1-c1ncc(Cl)cc1Cl</smiles>

SYP-9080

Fig. 1 The structures of commercial insecticides containing the substructures of diacylhydrazine and 3-bromo-1-(3-chloropyridin-2-yl)-1H-pyrazole

high insecticidal selectivity, and a large number of insecticidal molecules were discovered [8-23].

3-Bromo-1-(3-chloropyridin-2-yl)- $1 H$-pyrazole is an important scaffold and appear in several commercial insecticides structures, such as chlorantraniliprole [24], cyantraniliprole [25], and SYP-9080 (Fig. 1) [26]. In recent years, a large number of insecticidal molecules containing a 3-bromo-1-(3-chloropyridin-2-yl)-1 $H$-pyrazole were reported [27-30]. Among which, some diacylhydrazines containing 3-bromo-1-(3-chloropyridin-2-yl)$1 H$-pyrazole scaffold were also reported [11, 31], such as $\mathrm{N}$-(2-(2-(3-bromo-1-(3-chloropyridin-2-yl)- $1 \mathrm{H}$-pyrazole5-carbonyl)-2-(tert-butyl) hydrazinecarbonyl)-5-chloro3-methylphenyl) acetamide show 100\% larvicidal activity against Mythimna separate at $100 \mathrm{mg} \mathrm{L}^{-1}$. And in our previous works [15, 32-35], a series of diacylhydrazine derivatives containing 3-bromo-1-(3-chloropyridin2-yl)- $1 H$-pyrazole was also been confirmed to show good insecticidal activities.

Encouraged by descriptions above and as a continuation of insecticidal molecules with 3-bromo-1-(3chloropyridin-2-yl)- $1 H$-pyrazole, we herein sought to retain the substructure of 3-bromo-1-(3-chloropyridin2 -yl)- $1 H$-pyrazole and tert-butyl diacylhydrazine, and introducing different substituted aryls (Fig. 2). A series of novel diacylhydrazine derivatives was designed and synthesized. Structures of the synthesized compounds were characterized by ${ }^{1} \mathrm{H}$ NMR, ${ }^{13} \mathrm{C}$ NMR, and HR-MS. Results of bioassays indicated that most synthesized compounds exhibit good insecticidal activities against $P$. xylostella. In particular, the compounds $10 \mathrm{~g}, 10 \mathrm{~h}$, and $10 x$ exhibited excellent insecticidal activities, with $\mathrm{LC}_{50}$ values of 27.49 , 23.67, and $28.90 \mathrm{mg} \mathrm{L}^{-1}$, respectively. These compounds showed slightly higher insecticidal activity than commercial tebufenozide $\left(\mathrm{LC}_{50}=37.77 \mathrm{mg} \mathrm{L}^{-1}\right)$.

\section{Results and discussion}

\section{Chemistry}

The synthesis of the 3-bromo-1-(3-chloropyridin-2-yl)$1 \mathrm{H}$-pyrazole-5-carbohydrazide derivatives are depicted in Scheme 1. Firstly, the key intermediate 3-bromo-1-(3chloropyridin-2-yl)-1H-pyrazole-5-carboxylic acid (5) was obtained in good yield via reactions of hydrazinolysis, cyclization, bromination, oxydehydrogenation, and acidolysis by employing 2,3-dichloropyridine (1), hydrazine hydrate and diethyl maleate as starting materials $[24,33,34]$. Then compound 5 was allowed to further react with thionyl chloride under reflux to afford 

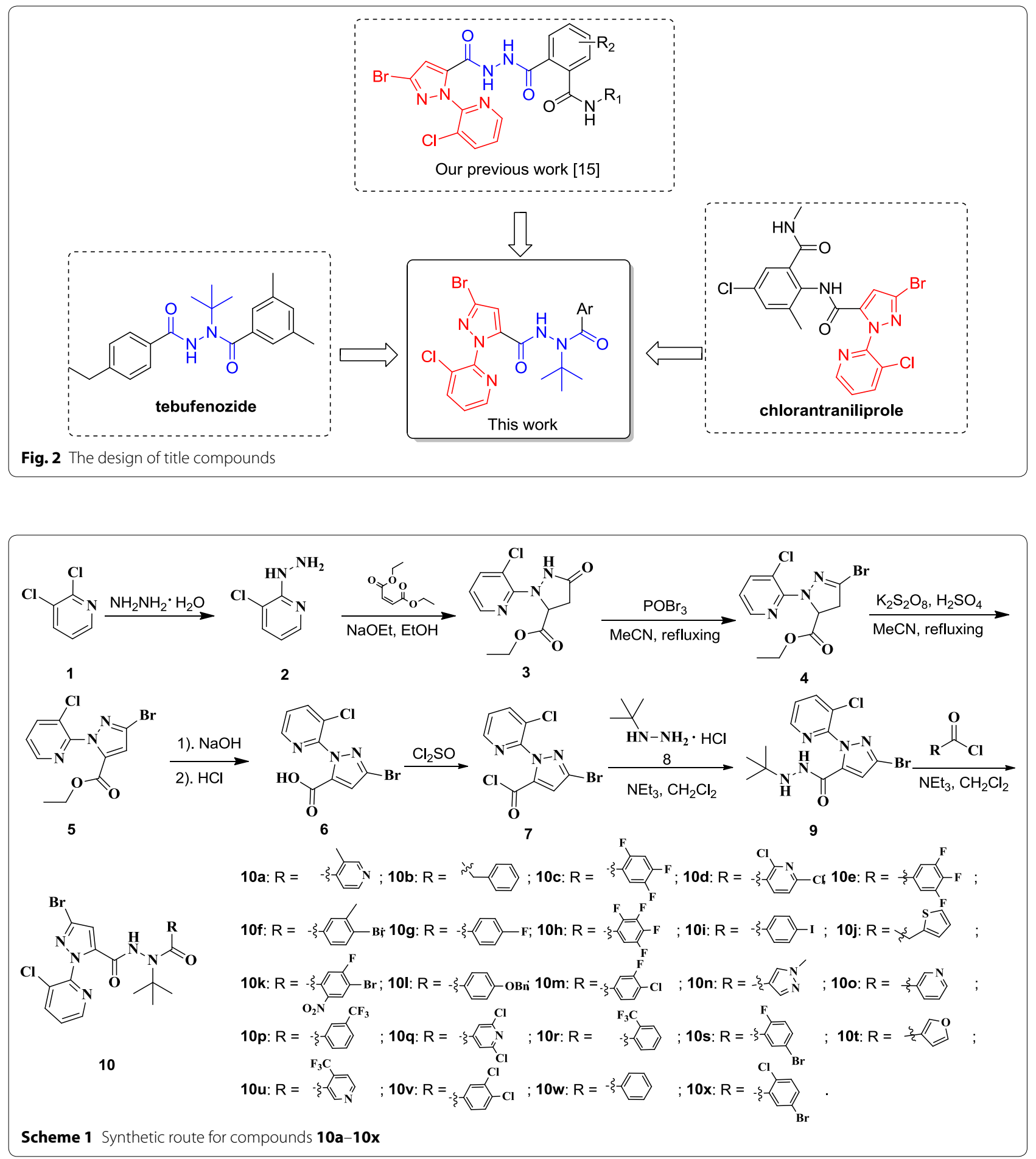

3-bromo-1-(3-chloropyridin-2-yl)-1H-pyrazole-5-carbonyl chloride (7) [35]. Subsequent treatment of intermediate 7 , with tert-butyl hydrazine hydrochloride (8) in the presence of triethylamine in trichloromethane at ambient temperature afforded 3-bromo- $N^{\prime}$-(tert-butyl)1-(3-chloropyridin-2-yl)-1H-pyrazole-5-carbohydrazide (9) in $80 \%$ yield. Finally, the title compounds $(10 a-10 x)$ were conveniently obtained in an $>70 \%$ yield by treating of 
intermediate 9 with the corresponding acyl chloride in the presence of triethylamine in acetone or acetonitrile.

Structures of the title compounds $(10 a-10 x)$ were established on basis of their spectroscopic data. In the ${ }^{1} \mathrm{H}$ NMR spectra, the $\mathrm{N}-\mathrm{H}$ proton appeared as a broad singlet near $\delta 11.10 \mathrm{ppm}$. The proton at position 5 of pyridine appeared as a doublet of doublets near $\delta 8.45$ due to the coupling coefficients from the protons at 3 and 4 positions of the pyridine ring; the coupling constants were ${ }^{3} J=4.7 \mathrm{~Hz}$ and ${ }^{4} J=1.5 \mathrm{~Hz}$ respectively. As well as the protons at positions 3 and 4 showed as doublet of doublets near $\delta 8.2$ and $7.7 \mathrm{ppm}$, respectively, because of the coupling coefficients from both 5 positions and the each other from 4 and 3 positions of the pyridine ring, respectively. 4-pyrazole-H exhibited a singlet near $\delta 6.90 \mathrm{ppm}$. The rest of the aromatic protons appeared range from 7.0 to $8.0 \mathrm{ppm}$, the nine protons $\left(-\mathrm{CH}_{3}\right)_{3}$ appeared as a singlet near $\delta 1.45 \mathrm{ppm}$; In ${ }^{13} \mathrm{C}$ NMR spectra for the fluorine contained compounds, the carbons were split into multiplet due to the coupling coefficients from " $\mathrm{F}$ ", take compound $10 \mathrm{~m}$ as example, the carbon near " $\mathrm{F}$ " resonance frequency is near $\delta_{C} 158.27 \mathrm{ppm}$ as a doublet and with the coupling constant $\left({ }^{1} J_{C-F}\right)$ was $249.5 \mathrm{~Hz}$; and the carbons at ortho-position of $\mathrm{F}$ were also split into doublets with coupling constant $\left({ }^{2} J_{C-F}\right)$ ranged from $18.1 \mathrm{~Hz}$ to $21.4 \mathrm{~Hz}$. The properties, ${ }^{1} \mathrm{H}$ NMR, ${ }^{13} \mathrm{C}$ NMR, ${ }^{19} \mathrm{~F}$ NMR, and HR-MS data of the synthesized compounds 10a to 10x are summarized in more detail in the "Experimental section".

\section{Insecticidal activity}

The insecticidal activities of the synthesized compounds against both Helicoverpa armigera and Plutella xylostella were evaluated using procedures reported previously [17, 33-36] and summarized in Tables 1 and 2, respectively. Commercial tebufenozide, chlorantraniliprole, and chlorpyrifos were used as positive controls.

The results listed in Table 1 indicated that the synthesized compounds displayed weak to good larvicidal activity against Helicoverpa armigera at the test concentration. For example, the larvicidal activity of compounds $10 \mathrm{c}$ to 10j, 10l, 10o-10q, 10v, and 10w showed $>50 \%$ mortality on $H$. armigera at $500 \mathrm{mg} \mathrm{L}^{-1}$, and the larvicidal activity of $10 \mathrm{~g}, 10 \mathrm{~h}$, and $10 \mathrm{w}$ were $70.8,87.5$, and $79.2 \%$, respectively, whereas the concentration was $100 \mathrm{mg} \mathrm{L}^{-1}$, the mortalities of $H$. armigera for compounds $10 \mathrm{~h}$ and $10 \mathrm{w}$ were still $>50 \%$.

As shown in Table 2, the synthesized compounds shown larvicidal activity against Plutella xylostella, with mortality range from 6.7 to $100 \%$. And it can be seen that most of the synthesized compounds show over $60 \%$ activity at $500 \mathrm{mg} \mathrm{L}^{-1}$, and compounds $10 \mathrm{e}, 10 \mathrm{~g}$ to $10 \mathrm{j}$ and 10w displayed $>90 \%$ activities. In particular, compounds
Table 1 Larvicidal activity of compounds 10a-10s against Helicoverpa armigera

\begin{tabular}{|c|c|c|c|c|c|}
\hline \multirow[t]{2}{*}{ Compounds } & \multicolumn{5}{|c|}{$\begin{array}{l}\text { Larvicidal activity (\%) at different } \\
\text { concentrations }\left(\mathrm{mg} \mathrm{L}^{-1}\right)\end{array}$} \\
\hline & 500 & 200 & 100 & 50 & 25 \\
\hline $10 a$ & 45.8 & 22.2 & 0.0 & / & / \\
\hline $10 \mathrm{~b}$ & 16.7 & 0.0 & / & / & / \\
\hline $10 c$ & 62.5 & 44.4 & 21.4 & 6.7 & / \\
\hline 10d & 58.3 & 38.9 & 14.3 & / & / \\
\hline $10 e$ & 62.5 & 44.4 & 21.4 & / & / \\
\hline $10 f$ & 58.3 & 38.9 & 14.3 & I & I \\
\hline $10 \mathrm{~g}$ & 70.8 & 55.6 & 35.7 & / & / \\
\hline $10 \mathrm{~h}$ & 87.5 & 77.8 & 64.3 & 43.3 & 16.7 \\
\hline $10 \mathrm{i}$ & 54.2 & 33.3 & 7.1 & / & / \\
\hline 10j & 66.7 & 40.0 & 28.6 & 13.3 & / \\
\hline 10k & 33.3 & 5.6 & 0.0 & / & I \\
\hline 101 & 58.3 & 38.9 & 14.3 & / & / \\
\hline $10 \mathrm{~m}$ & 37.5 & 11.1 & 0.0 & / & / \\
\hline $10 n$ & 41.7 & 16.7 & 0.0 & / & / \\
\hline 100 & 63.3 & 46.7 & 26.7 & 6.7 & / \\
\hline $10 p$ & 54.2 & 33.3 & 7.1 & / & / \\
\hline $10 q$ & 58.3 & 38.9 & 14.3 & / & / \\
\hline $10 r$ & 30.0 & 0.0 & / & / & I \\
\hline $10 \mathrm{~s}$ & 41.7 & 16.7 & 0.0 & / & / \\
\hline $10 t$ & 33.3 & 5.6 & 0.0 & / & / \\
\hline $10 u$ & 0.0 & / & / & I & / \\
\hline $10 v$ & 54.2 & 33.3 & 7.0 & / & I \\
\hline $10 w$ & 79.2 & 60.0 & 53.3 & 23.3 & 6.7 \\
\hline $10 x$ & 41.7 & 16.7 & 0.0 & / & / \\
\hline Tebufenozide & 100 & 93.3 & 70.0 & 50 & 40.0 \\
\hline Chlorpyrifos & 100 & 100 & 100 & 90 & 83 \\
\hline Chlorantraniliprole & 100 & 100 & 100 & 100 & 100 \\
\hline
\end{tabular}

$10 \mathrm{~g}, 10 \mathrm{~h}$ and 10w showed good larvicidal activity, both 10h and 10w showed 100\% activities against Plutella xylostella at $200 \mathrm{mg} \mathrm{L}^{-1}$, and the activity of compound $10 \mathrm{~g}$ was up to $96.7 \%$. When the concentration was $50 \mathrm{mg} \mathrm{L}^{-1}$, the activities of compounds $10 \mathrm{~g}, 10 \mathrm{~h}$ and 10w were $66.7,76.7$ and $70 \%$ at $50 \mathrm{mg} \mathrm{L}^{-1}$, respectively, whereas these three compounds showed moderate activity at $25 \mathrm{mg} \mathrm{L}^{-1}$.

The median lethal concentrations $\left(\mathrm{LC}_{50}\right)$ of compounds $10 \mathrm{c}, 10 \mathrm{e}, 10 \mathrm{~g}, 10 \mathrm{~h}, 10 \mathrm{i}, 10 \mathrm{j}$ and $10 \mathrm{w}$ were further determined. For comparison, the $\mathrm{LC}_{50}$ value of tebufenozide (a commonly used insecticide) were also evaluated. The results are given in Table 3. The $\mathrm{LC}_{50}$ values of compounds $10 \mathrm{e}, 10 \mathrm{~g}, 10 \mathrm{~h}, 10 \mathrm{j}$ and $10 \mathrm{w}$ were less than $100 \mathrm{mg} \mathrm{L}^{-1}$ (Table 3). In particular, the compounds $10 \mathrm{~g}, 10 \mathrm{~h}$, and $10 \mathrm{w}$ exhibited excellent insecticidal activities, with $\mathrm{LC}_{50}$ values of $27.49,23.67$, and $28.90 \mathrm{mg} \mathrm{L}^{-1}$, 
Table 2 Larvicidal activity of compounds (10a-10s) against Plutella xylostella

\begin{tabular}{|c|c|c|c|c|c|}
\hline \multirow[t]{2}{*}{ Compounds } & \multicolumn{5}{|c|}{$\begin{array}{l}\text { Larvicidal activity (\%) at different } \\
\text { concentrations }\left(\mathrm{mg} \mathrm{L}^{-1}\right)\end{array}$} \\
\hline & 500 & 200 & 100 & 50 & 25 \\
\hline $10 a$ & 70.0 & 46.7 & 21 & / & / \\
\hline $10 \mathrm{~b}$ & 33.3 & 16.7 & 0.0 & / & / \\
\hline $10 c$ & 86.7 & 56.7 & 30.0 & 16.7 & / \\
\hline $10 d$ & 76.7 & 53.3 & 23.6 & / & / \\
\hline $10 e$ & 90.0 & 73.3 & 53.3 & 36.7 & 16.7 \\
\hline $10 f$ & 66.7 & 53.5 & 30.2 & / & / \\
\hline $10 \mathrm{~g}$ & 100 & 96.7 & 80.0 & 66.7 & 50.0 \\
\hline $10 \mathrm{~h}$ & 100 & 100 & 93.3 & 76.7 & 53.3 \\
\hline $10 \mathrm{i}$ & 90.0 & 63.3 & 43.3 & 33.3 & 16.7 \\
\hline $10 \mathrm{j}$ & 96.7 & 83.3 & 53.3 & 36.7 & 23.3 \\
\hline $10 k$ & 56.7 & 23.3 & 3.3 & / & / \\
\hline 101 & 73.3 & 53.3 & 16.7 & 6.7 & / \\
\hline $10 \mathrm{~m}$ & 63.3 & 33.3 & 16.7 & / & / \\
\hline $10 n$ & 56.7 & 33.3 & 13.1 & / & / \\
\hline 100 & 80.0 & 63.3 & 33.7 & 16.7 & / \\
\hline $10 p$ & 76.7 & 53.3 & 13.0 & / & / \\
\hline $10 q$ & 73.3 & 49.0 & 20.0 & / & / \\
\hline $10 e$ & 43.3 & 23.3 & 13.3 & / & / \\
\hline $10 \mathrm{~s}$ & 66.7 & 33.3 & 16.7 & / & / \\
\hline $10 t$ & 43.3 & 23.3 & 6.7 & / & / \\
\hline $10 u$ & 6.7 & 0.0 & / & / & / \\
\hline $10 v$ & 80.0 & 66.7 & 23.3 & / & / \\
\hline $10 w$ & 100 & 100 & 86.7 & 70.0 & 46.7 \\
\hline $10 x$ & 66.7 & 33.3 & 13.3 & / & / \\
\hline Tebufenozide & 100 & 96.7 & 80.0 & 56.7 & 26.7 \\
\hline Chlorpyrifos & 100 & 100 & 100 & 90 & 83 \\
\hline Chlorantraniliprole & 100 & 100 & 100 & 100 & 100 \\
\hline
\end{tabular}

Table 3 LC $_{50}$ values for insecticidal activity against Plutella xylostella

\begin{tabular}{llll}
\hline Comp. & $\mathbf{y}=\mathbf{a}+\mathbf{b x}$ & $\mathbf{r}$ & $\mathbf{L C}_{\mathbf{5 0}}\left(\mathbf{m g ~ L}^{-\mathbf{1}}\right)$ \\
\hline $\mathbf{1 0 c}$ & $Y=0.632181+1.993794 x$ & 0.99 & 155.13 \\
$\mathbf{1 0 e}$ & $Y=1.699094+1.701997 x$ & 0.99 & 86.98 \\
$\mathbf{1 0 g}$ & $Y=2.248458+1.91187 x$ & 0.97 & 27.49 \\
$\mathbf{1 0 h}$ & $Y=1.687545+2.410609 x$ & 0.99 & 23.67 \\
$\mathbf{1 0 i}$ & $Y=1.661246+1.658921 x$ & 0.98 & 102.95 \\
$\mathbf{1 0 j}$ & $Y=1.699094+1.701997 x$ & 0.99 & 69.07 \\
$\mathbf{1 0 w}$ & $Y=1.85713+2.15129 x$ & 0.99 & 28.90 \\
Tebufenozide & $Y=1.429139+2.2641 x$ & 0.99 & 37.77 \\
\hline
\end{tabular}

respectively. These compounds showed slightly higher insecticidal activity than commercial tebufenozide $\left(\mathrm{LC}_{50}=37.77 \mathrm{mg} \mathrm{L}^{-1}\right)$. As revealed by data in Tables 1 and 2 , the insecticidal activity of the title compound was effected by $\mathrm{R}$ group. When $\mathrm{R}$ was a benzene ring (10w), the compound showed excellent insecticidal activity (compare with tebufenozide), and the activity could be slightly enhanced by introduction of a fluorine at 4 position of benzene (compound $\mathbf{1 0 g}$ ) or four fluorines on benzene (10h). However, the activity decreased when benzene was substituted by tri-fluorine at $3,4,5$ positions, as well as decreased by introducing other substituents, such as nitro, 2-trifluoromethyl, 3-trifluoromethyl, 3,4-di-chloro, and 4-iodine. In addition, when $\mathrm{R}$ was a heterocyclic ring (i.e., pyridine, pyrazole, furan), the corresponding compounds showed much weaker activities than the compounds with a benzene ring. Moreover, a compound containing the benzyl show no larvicidal activity. But interestingly, a compound containing the 2-thiophen-2-yl (10j) was found to show good insecticidal activity.

\section{Experimental section}

Materials and instruments

All aromatic acids were purchased from Accela ChemBio Co., Ltd (Shanghai, China). Melting points were determined using a XT-4 binocular microscope (Beijing Tech Instrument Co., China) and left uncorrected. The NMR spectra was recorded on a AVANCE III HD 400M NMR (Bruker corporation, Switzerland) or JEOL ECX 500 NMR spectrometer (JEOL Ltd., Japan) operating at room temperature using DMSO as solvent. HR-MS was recorded on an Orbitrap LC-MS instrument (Q-Exative, Thermo Scientific ${ }^{\mathrm{TM}}$, American). The course of the reactions was monitored by TLC; analytical TLC was performed on silica gel GF254. All reagents were of analytical grade or chemically pure. All anhydrous solvents were dried and purified according to standard techniques just before use.

\section{Synthetic procedures}

General procedure for intermediates (2-6)

Intermediates 2-6 were prepared by following the known procedures, [24, 33, 34] and the acyl chloride (7) was synthesized according to reported method [35]. The detailed synthetic procedures and physical properties for these intermediates can be found in Additional file 1.

\section{Synthesis of intermediate (9)}

To a well-stirred suspension of tert-butyl hydrazine hydrochloride $\mathbf{8}$ in dichloromethane, two equivalents of triethylamine was added, the resulted mixture was stirred at room temperature for $10 \mathrm{~min}$, then the solution of acyl chloride 7 in dichloromethane was then added dropwise. After stirring and refluxing for $2 \mathrm{~h}$, dichloromethane was removed in vacuo. The mixture was washed with saturated sodium bicarbonate solution. The solution was 
filtered to obtain a crude product, which was recrystallized with ethanol to obtain the 3-bromo- $N^{\prime}$-(tert-butyl)1-(3-chloropyridin-2-yl)-1H-pyrazole-5-carbohydrazide (9). Brown solid, yield, $80 \%,{ }^{1} \mathrm{H}$ NMR $(500 \mathrm{MHz}$, DMSOD6) $\delta 10.08$ (brs, $1 \mathrm{H}, \mathrm{N}-\mathrm{H}), 8.47$ (d, $J=4.6 \mathrm{~Hz}, 1 \mathrm{H}$, pyridine-H), $8.15(\mathrm{~d}, J=8.0 \mathrm{~Hz}, 1 \mathrm{H}$, pyridine-H), 7.58 (dd, $J=8.0,4.7 \mathrm{~Hz}, 1 \mathrm{H}$, pyridine $-\mathrm{H}), 7.25(\mathrm{~s}, 1 \mathrm{H}$, pyrazole- $\mathrm{H})$, 4.78 (brs, $1 \mathrm{H}, \mathrm{N}-\mathrm{H}), 0.96$ (s, 9H, $\left.3 \mathrm{CH}_{3}\right)$.

\section{General procedure for the preparation of title compounds (10a-10y)}

Different fresh acyl chloride $(1 \mathrm{mmol})$ were added to a well-stirred solution of 9 ( $1 \mathrm{mmol})$ in chloroform $(5 \mathrm{~mL})$ in present of triethylamine. The resulting mixture was stirred for $50 \mathrm{~min}$ at ambient temperature to afford a white solid, and then filtered and recrystallized from ethanol in good yield.

N'-(3-Bromo-1-(3-chloropyridin-2-yl)-1H-pyrazole-5-carbony I)-N-(tert-butyl)-3-methylisonicotinohydrazide (10a)

White solid. M.p: $286-287{ }^{\circ} \mathrm{C}$; yield: $78 \%$; ${ }^{1} \mathrm{H}$ NMR (400 MHz, DMSO) $\delta 10.98$ (s, 1H, N-H), 8.50 (dd, ${ }^{3} J=4.7 \mathrm{~Hz},{ }^{4} J=1.5 \mathrm{~Hz}, 1 \mathrm{H}$, pyridine- $\left.\mathrm{H}\right), 8.44(\mathrm{~s}, 1 \mathrm{H}$, pyridine-H), 8.35 (d, $\left.{ }^{3} J=4.9 \mathrm{~Hz}, 1 \mathrm{H}, \mathrm{Ar}-\mathrm{H}\right), 8.23$ (dd, ${ }^{3} J=8.1 \mathrm{~Hz},{ }^{4} J=1.5 \mathrm{~Hz}, 1 \mathrm{H}$, pyridine-H), 7.67 $\left(\mathrm{dd},{ }^{3} J=8.1 \mathrm{~Hz},{ }^{4} J=4.7 \mathrm{~Hz}, 1 \mathrm{H}\right.$, pyridine-H), 6.97 (s, $1 \mathrm{H}$, pyrazole- $\mathrm{H}), 6.69(\mathrm{~s}, 1 \mathrm{H}$, pyridine- $\mathrm{H}), 2.17$ (s, $\left.3 \mathrm{H},-\mathrm{CH}_{3}\right), 1.45\left(\mathrm{~s}, 9 \mathrm{H}, 3 \mathrm{CH}_{3}\right) ;{ }^{13} \mathrm{C} \mathrm{NMR}(100 \mathrm{MHz}$, DMSO) $\delta 170.00,157.50,151.54,147.99,147.70$, 147.02 , 144.56, 140.09, 137.31, 128.01, 127.45, 127.25, 119.22, 110.78, 61.57, 27.66, 15.68. HR-MS $\left(\mathrm{ESI}^{+}\right) \mathrm{m} / z$ Calcd for $\mathrm{C}_{20} \mathrm{H}_{20} \mathrm{BrClN}_{6} \mathrm{O}_{2}[\mathrm{M}+\mathrm{H}]^{+}$491.05978; found 491.05980 .

\section{3-Bromo-N'-(tert-butyl)-1-(3-chloropyridin-2-yl)-N'-(2-phenyl acetyl)-1H-pyrazole-5-carbohydrazide (10b)}

White solid, M.p: $211-213{ }^{\circ} \mathrm{C}$; yield: $83 \%$; ${ }^{1} \mathrm{H}$ NMR (400 MHz, DMSO) $\delta 11.10$ (s, 1H, N-H), 8.49 (dd, ${ }^{3} J=4.7 \mathrm{~Hz},{ }^{4} J=1.5 \mathrm{~Hz}, 1 \mathrm{H}$, pyridine-H), 8.27 (dd, ${ }^{3} J=8.1 \mathrm{~Hz},{ }^{4} J=1.5 \mathrm{~Hz}, 1 \mathrm{H}$, pyridine-H), $7.68(\mathrm{dd}$, ${ }^{3} J=8.1 \mathrm{~Hz},{ }^{4} J=4.7 \mathrm{~Hz}, 1 \mathrm{H}$, pyridine- $\left.\mathrm{H}\right), 7.31(\mathrm{~s}, 1 \mathrm{H}$, benzene-H), 7.30-7.19 (m, 3H, benzene-H), 7.12-7.07 (m, 2H, benzene-H), $4.04\left(\mathrm{~s}, 2 \mathrm{H},-\mathrm{CH}_{2}-\right), 1.33(\mathrm{~s}, 9 \mathrm{H}$, $\left.3 \mathrm{CH}_{3}\right) ;{ }^{13} \mathrm{C}$ NMR (100 MHz, DMSO) $\delta$ 172.28, 157.85, $150.97,147.72,140.25,137.77,135.92,129.97,128.59$, 127.96, 127.42, 126.82, 123.46, 111.46, 61.06, 40.94, 27.87. HR-MS $\left(\mathrm{ESI}^{+}\right) \mathrm{m} / z$ Calcd for $\mathrm{C}_{21} \mathrm{H}_{21} \mathrm{BrClN}_{5} \mathrm{O}_{2}[\mathrm{M}+\mathrm{H}]^{+}$ 490.06399; found 490.06392.

3-Bromo-N'-(tert-butyl)-1-(3-chloropyridin-2-yl)- $N^{\prime}-(2,4,5-$ tri fluorobenzoyl)-1H-pyrazole-5-carbohydrazide (10c)

White solid, M.p: $226-227{ }^{\circ} \mathrm{C}$; yield: $85 \%$; ${ }^{1} \mathrm{H}$ NMR (400 MHz, DMSO) $\delta 11.18(\mathrm{~s}, 1 \mathrm{H}, \mathrm{N}-\mathrm{H}), 8.45$ (dd,
${ }^{3} J=4.7 \mathrm{~Hz},{ }^{4} J=1.5 \mathrm{~Hz}, 1 \mathrm{H}$, pyridine-H), $8.19(\mathrm{dd}$, ${ }^{3} J=8.1 \mathrm{~Hz},{ }^{4} J=1.5 \mathrm{~Hz}, 1 \mathrm{H}$, pyridine $\left.-\mathrm{H}\right), 7.67(\mathrm{dd}$, ${ }^{3} J=8.1 \mathrm{~Hz},{ }^{4} J=4.7 \mathrm{~Hz}, 1 \mathrm{H}$, pyridine-H), 7.65-7.59 (m, $1 \mathrm{H}$, benzene-H), $7.20\left(\mathrm{td},{ }^{3} J=9.4 \mathrm{~Hz},{ }^{4} J=6.3 \mathrm{~Hz}, 1 \mathrm{H}\right.$, benzene-H), 7.03 (s, $1 \mathrm{H}$, pyrazole- $\mathrm{H}), 1.42\left(\mathrm{~s}, 9 \mathrm{H}, 3 \mathrm{CH}_{3}\right)$; ${ }^{19} \mathrm{~F}$ NMR (471 MHz, DMSO-D6) $\delta-116.38,-132.12$; ${ }^{13} \mathrm{C}$ NMR (100 MHz, DMSO) $\delta 165.61,163.14(\mathrm{~d}$, $J=229.6 \mathrm{~Hz}), 157.08,153.64(\mathrm{~d}, J=243.2 \mathrm{~Hz}), 148.14$, $147.62,139.98,136.94,128.10,127.49,127.36,122.50(\mathrm{dd}$, $J=20.0,4.3 \mathrm{~Hz}), 111.11,116.74(\mathrm{dd}, J=20.8,5.8 \mathrm{~Hz})$, $106.83(\mathrm{dd}, J=28.6,21.8 \mathrm{~Hz}) 61.97,27.66$; HR-MS $\left(\mathrm{ESI}^{+}\right)$ $m / z$ Calcd for $\mathrm{C}_{20} \mathrm{H}_{16} \mathrm{BrClF}_{3} \mathrm{~N}_{5} \mathrm{O}_{2}[\mathrm{M}+\mathrm{H}]^{+} 530.02008$; found 530.02012 .

$N^{\prime}$-(3-Bromo-1-(3-chloropyridin-2-yl)-1H-pyrazole-5-carbony l)-N-(tert-butyl)-2,6-dichloronicotinohydrazide (10d)

White solid. M.p: 223-224 ${ }^{\circ} \mathrm{C}$; yield: $65 \%$; ${ }^{1} \mathrm{H}$ NMR (400 MHz, DMSO) $\delta 11.20(\mathrm{~s}, 1 \mathrm{H}, \mathrm{N}-\mathrm{H}), 8.50(\mathrm{~d}$, ${ }^{3} J=3.5 \mathrm{~Hz}, 1 \mathrm{H}$, pyridine-H), $8.21\left(\mathrm{dd},{ }^{3} J=8.1 \mathrm{~Hz}\right.$, ${ }^{4} J=1.4 \mathrm{~Hz}, 1 \mathrm{H}$, pyridine-H), $7.68\left(\mathrm{dd},{ }^{3} J=8.1 \mathrm{~Hz}\right.$, ${ }^{4} J=4.7 \mathrm{~Hz}, 1 \mathrm{H}$, pyridine- $\left.\mathrm{H}\right), 7.56(\mathrm{~s}, 1 \mathrm{H}$, pyridine- $\mathrm{H})$, 7.55 (s, 1H, pyridine-H), 6.99 (s, 1H, pyrazole-H), 1.44 $\left(\mathrm{s}, 9 \mathrm{H}, 3 \mathrm{CH}_{3}\right) .{ }^{13} \mathrm{C} \mathrm{NMR}(100 \mathrm{MHz}, \mathrm{DMSO}) \delta 166.76$, $166.00,165.37,149.28,148.40,148.00,147.98,147.73$, $140.17,140.14,139.45,136.93,136.91,127.96,127.53$, 127.37, 123.72, 111.42, 62.07, 27.51; HR-MS $\left(\mathrm{ESI}^{+}\right) \mathrm{m} / z$ Calcd for $\mathrm{C}_{19} \mathrm{H}_{16} \mathrm{BrCl}_{3} \mathrm{~N}_{6} \mathrm{O}_{2},[\mathrm{M}+\mathrm{H}]^{+}$544.96565; found 544.96531; $[\mathrm{M}+\mathrm{Na}]^{+} 566.94759$; found 566.94752 .

\section{3-Bromo-N'-(tert-butyl)-1-(3-chloropyridin-2-yl)- $N^{\prime}-(3,4,5-$ trif} luorobenzoyl)-1H-pyrazole-5-carbohydrazide (10e)

White solid. M.p: 260-262; yield: $73 \%$; ${ }^{1} \mathrm{H}$ NMR (400 MHz, DMSO) $\delta 11.13(\mathrm{~s}, 1 \mathrm{H}, \mathrm{N}-\mathrm{H}), 8.42$ (dd, ${ }^{3} J=4.7 \mathrm{~Hz},{ }^{4} J=1.5 \mathrm{~Hz}, 1 \mathrm{H}$, pyridine-H), $8.18(\mathrm{dd}$, ${ }^{3} J=8.1 \mathrm{~Hz},{ }^{4} J=1.5 \mathrm{~Hz}, 1 \mathrm{H}$, pyridine-H), $7.66(\mathrm{dd}$, ${ }^{3} J=8.1 \mathrm{~Hz},{ }^{4} J=4.7 \mathrm{~Hz}, 1 \mathrm{H}$, pyridine-H), 7.31-7.23 (m, $2 \mathrm{H}$, benzene-H), 7.05 (s, 1H, pyrazole- $\mathrm{H}), 1.41(\mathrm{~s}, 9 \mathrm{H}$, $\left.3 \mathrm{CH}_{3}\right) ;{ }^{19} \mathrm{~F}$ NMR (471 MHz, DMSO-D6) $\delta-116.37$, -132.12, -142.79; ${ }^{13} \mathrm{C}$ NMR (100 MHz, DMSO) $\delta$ 168.68, $156.82(\mathrm{~d}, J=245 \mathrm{~Hz}), 151.24(\mathrm{~d}, J=9.7 \mathrm{~Hz})$ $148.08(\mathrm{~d}, J=245 \mathrm{~Hz}), 147.55,139.95,137.11,128.11$, $127.50,127.46,112.58,112.36,111.01,100.00,61.78$, 27.61; HR-MS $\left(\mathrm{ESI}^{+}\right) \mathrm{m} / z$ Calcd for $\mathrm{C}_{20} \mathrm{H}_{16} \mathrm{BrClF}_{3} \mathrm{~N}_{5} \mathrm{O}_{2}$, $[\mathrm{M}+\mathrm{H}]^{+}$530.02008; found 530.02013; $[\mathrm{M}+\mathrm{Na}]^{+}$ 552.00202 , found 552.00243 .

3-Bromo-N'-(4-bromo-3-methylbenzoyl)-N'-(tert-butyl)-1-(3chloropyridin-2-yl)-1H-pyrazole-5-carbohydrazide (10f) White solid. M.p: $262-263{ }^{\circ} \mathrm{C}$; yield: $72 \%$; ${ }^{1} \mathrm{H}$ NMR (400 MHz, DMSO) $\delta 10.88(\mathrm{~s}, 1 \mathrm{H}, \mathrm{N}-\mathrm{H}), 8.53-8.44$ $(\mathrm{m}, 1 \mathrm{H}, \mathrm{Ar}-\mathrm{H}), 8.27-8.15(\mathrm{~m}, 1 \mathrm{H}, \mathrm{Ar}-\mathrm{H}), 7.67(\mathrm{dd}$, ${ }^{3} J=12.2 \mathrm{~Hz},{ }^{4} J=7.3 \mathrm{~Hz}, 1 \mathrm{H}$, pyridine-H), 7.52-7.41 (m, $1 \mathrm{H}, \mathrm{Ar}-\mathrm{H}), 7.33$ (s, 1H, Ar-H), 6.98 (s, 1H, pyrazole-H), 
$6.70\left(\mathrm{~d},{ }^{3} \mathrm{~J}=16.0 \mathrm{~Hz}, 1 \mathrm{H}, \mathrm{Ar}-\mathrm{H}\right), 2.17\left(\mathrm{~s}, 3 \mathrm{H}, \mathrm{CH}_{3}\right), 1.44$ (s, 9H, $\left.3 \mathrm{CH}_{3}\right) \cdot{ }^{13} \mathrm{C}$ NMR (100 MHz, DMSO) $\delta 171.28$, 157.32 , 147.98, 147.63, 140.04, 137.60, 133.14, 128.19, 127.96, 127.41, 127.20, 121.90, 110.79, 61.30, 27.76, 18.63; HR-MS $\left(\mathrm{ESI}^{+}\right) \mathrm{m} / z$ Calcd for $\mathrm{C}_{21} \mathrm{H}_{20} \mathrm{Br}_{2} \mathrm{ClN}_{5} \mathrm{O}_{2}$, $[\mathrm{M}+\mathrm{H}]^{+}$567.97450; found 567.97471.

\section{3-Bromo-N'-(tert-butyl)-1-(3-chloropyridin-2-yl)-N'-(4-fluoro benzoyl)-1H-pyrazole-5-carbohydrazide (10g)}

White solid, M.p: $256-257{ }^{\circ} \mathrm{C}$; yield: $82 \%$; ${ }^{1} \mathrm{H}$ NMR (400 MHz, DMSO) $\delta 11.04(\mathrm{~s}, 1 \mathrm{H}, \mathrm{N}-\mathrm{H}), 8.45(\mathrm{dd}$, ${ }^{3} J=4.7 \mathrm{~Hz},{ }^{4} J=1.4 \mathrm{~Hz}, 1 \mathrm{H}$, pyridine-H), $8.17(\mathrm{dd}$, ${ }^{3} J=8.1 \mathrm{~Hz},{ }^{4} J=1.4 \mathrm{~Hz}, 1 \mathrm{H}$, pyridine-H), $7.63(\mathrm{dd}$, ${ }^{3} J=8.1 \mathrm{~Hz},{ }^{4} J=4.7 \mathrm{~Hz}, 1 \mathrm{H}$, pyridine- $\left.\mathrm{H}\right), 7.46-7.37(\mathrm{~m}$, $2 \mathrm{H}$, benzene-H), $7.19\left(\mathrm{t},{ }^{3} J=8.9 \mathrm{~Hz}, 2 \mathrm{H}\right.$, benzene- $\left.\mathrm{H}\right)$, $6.90(\mathrm{~s}, 1 \mathrm{H}$, pyrazole- $\mathrm{H}), 1.41\left(\mathrm{~s}, 9 \mathrm{H}, 3 \mathrm{CH}_{3}\right) ;{ }^{19} \mathrm{~F}$ NMR (471 MHz, DMSO-D6) $\delta-110.71 ;{ }^{13} \mathrm{C}$ NMR $(100 \mathrm{MHz}$, DMSO) $\delta 170.98,164.36,\left(\mathrm{~d},{ }^{1} J_{\mathrm{C}-\mathrm{F}}=246.7 \mathrm{~Hz}\right), 156.79$, $148.08,147.62,139.95,137.58,133.68,129.89,129.81$, $127.92,127.33,115.24\left(\mathrm{~d},{ }^{2} J_{\mathrm{C}-\mathrm{F}}=21.7 \mathrm{~Hz}\right), 110.67,61.31$, 27.81; HR-MS $\left(\mathrm{ESI}^{+}\right) \mathrm{m} / z$ Calcd for $\mathrm{C}_{20} \mathrm{H}_{18} \mathrm{BrClFN}_{5} \mathrm{O}_{2}$, $[\mathrm{M}+\mathrm{H}]^{+}$494.03892, found 494.03852.

\section{3-Bromo-N'-(tert-butyl)-1-(3-chloropyridin-2-yl)- $N^{\prime}-(2,3,4,5$-tet rafluorobenzoyl)-1H-pyrazole-5-carbohydrazide (10 h)}

White solid, M.p: $185-187{ }^{\circ} \mathrm{C}$; yield: $69 \%$; ${ }^{1} \mathrm{H}$ NMR $(400 \mathrm{MHz}, \mathrm{DMSO}) \delta 11.24(\mathrm{~s}, 1 \mathrm{H}, \mathrm{N}-\mathrm{H}), 8.44(\mathrm{dd}$, ${ }^{3} J=4.7 \mathrm{~Hz},{ }^{4} J=1.5 \mathrm{~Hz}, 1 \mathrm{H}$, pyridine- $\left.\mathrm{H}\right), 8.20(\mathrm{dd}$, ${ }^{3} J=8.1,{ }^{4} J=1.5 \mathrm{~Hz}, 1 \mathrm{H}$, pyridine-H), $7.68\left(\mathrm{dd},{ }^{3} J=8.1\right.$, ${ }^{4} J=4.7 \mathrm{~Hz}, 1 \mathrm{H}$, pyridine- $\left.\mathrm{H}\right), 7.19-7.11(\mathrm{~m}, 1 \mathrm{H}$, benzene-H), 7.09 (s, $1 \mathrm{H}$, pyrazole- $\mathrm{H}), 1.43\left(\mathrm{~s}, 9 \mathrm{H}, 3 \mathrm{CH}_{3}\right)$; ${ }^{19} \mathrm{~F}$ NMR (471 MHz, DMSO-D6) $\delta-138.96,-141.16$, $-154.38, \quad-155.29 ;{ }^{13} \mathrm{C}$ NMR $(126 \mathrm{MHz}$, DMSOD6) $\delta 164.54,157.29,148.20,147.65,147.47-147.17$, $145.68-144.33$, 143.11-142.51, 141.91-140.72, 140.05, $139.83-139.15,136.84,128.23,127.61,127.50,110.55$ $(\mathrm{d}, J=20.3 \mathrm{~Hz}), 62.35,27.65$; HR-MS $\left(\mathrm{ESI}^{+}\right) \mathrm{m} / z$ Calcd for $\mathrm{C}_{20} \mathrm{H}_{15} \mathrm{BrClF}_{4} \mathrm{~N}_{5} \mathrm{O}_{2},[\mathrm{M}+\mathrm{H}]^{+}$548.01065, found 548.01032 .

\section{3-Bromo-N'-(tert-butyl)-1-(3-chloropyridin-2-yl)-N'-(4-iodobe} nzoyl)-1H-pyrazole-5-carbohydrazide (10i)

White solid. M.p: $268-269{ }^{\circ} \mathrm{C}$; yield: $76 \%$; ${ }^{1} \mathrm{H}$ NMR $(400 \mathrm{MHz}, \mathrm{DMSO}) \delta 11.05(\mathrm{~s}, 1 \mathrm{H}, \mathrm{N}-\mathrm{H}), 8.44(\mathrm{dd}$, ${ }^{3} J=4.7 \mathrm{~Hz},{ }^{4} J=1.5 \mathrm{~Hz}, 1 \mathrm{H}$, pyridine-H), $8.16(\mathrm{dd}$, ${ }^{3} J=8.1 \mathrm{~Hz},{ }^{4} J=1.5 \mathrm{~Hz}, 1 \mathrm{H}$, pyridine-H), $7.73(\mathrm{~d}$, ${ }^{3} J=8.4 \mathrm{~Hz}, 2 \mathrm{H}$, benzene- $\left.\mathrm{H}\right), 7.63\left(\mathrm{dd},{ }^{3} J=8.1 \mathrm{~Hz}\right.$, ${ }^{4} J=4.7 \mathrm{~Hz}, 1 \mathrm{H}$, pyridine- $\left.\mathrm{H}\right), 7.15\left(\mathrm{~d},{ }^{3} J=8.4 \mathrm{~Hz}, 2 \mathrm{H}\right.$, benzene- $\mathrm{H}), 6.90(\mathrm{~s}, 1 \mathrm{H}$, pyrazole- $\mathrm{H}), 1.41\left(\mathrm{~s}, 9 \mathrm{H}, 3 \mathrm{CH}_{3}\right)$; ${ }^{13} \mathrm{C}$ NMR (100 MHz, DMSO) $\delta 171.22,156.79,148.06$, 147.60, 139.96, 137.53, 136.98, 136.73, 129.23, 127.94, 127.34, 110.75, 97.17, 61.39, 27.77; HR-MS $\left(\mathrm{ESI}^{+}\right) \mathrm{m} / z$
Calcd for $\mathrm{C}_{20} \mathrm{H}_{18} \mathrm{BrClIN}_{5} \mathrm{O}_{2},[\mathrm{M}+\mathrm{H}]^{+}$601.94498, found 601.94452 .

3-Bromo-N'-(tert-butyl)-1-(3-chloropyridin-2-yl)-N'-(2-(thiop hen-2-yl)acetyl)-1H-pyrazole-5-carbohydrazide (10j)

White solid, M.p: $219-220{ }^{\circ} \mathrm{C}$; yield: $72 \% ;{ }^{1} \mathrm{H}$ NMR (400 MHz, DMSO) $\delta 11.13(\mathrm{~s}, 1 \mathrm{H}, \mathrm{N}-\mathrm{H}), 8.50(\mathrm{dd}$, ${ }^{3} J=4.7 \mathrm{~Hz},{ }^{4} J=1.5 \mathrm{~Hz}, 1 \mathrm{H}$, pyridine- $\left.\mathrm{H}\right), 8.27(\mathrm{dd}$, ${ }^{3} J=8.1 \mathrm{~Hz},{ }^{4} J=1.5 \mathrm{~Hz}, 1 \mathrm{H}$, pyridine-H), $7.67(\mathrm{dd}$, ${ }^{3} J=8.1 \mathrm{~Hz},{ }^{4} J=4.7 \mathrm{~Hz}, 1 \mathrm{H}$, pyridine-H), $7.39(\mathrm{dd}$, $\left.{ }^{3} J=5.1 \mathrm{~Hz},{ }^{4} J=1.2 \mathrm{~Hz}, 1 \mathrm{H}\right), 7.35(\mathrm{~s}, 1 \mathrm{H}$, pyrazoleH), $6.95\left(\mathrm{dd},{ }^{3} J=5.1 \mathrm{~Hz},{ }^{4} J=3.4 \mathrm{~Hz}, 1 \mathrm{H}\right), 6.83(\mathrm{dd}$, $\left.{ }^{3} J=3.4 \mathrm{~Hz},{ }^{4} J=1.0 \mathrm{~Hz}, 1 \mathrm{H}\right), 3.95\left(\mathrm{~d},{ }^{3} J=17.3 \mathrm{~Hz}\right.$, $1 \mathrm{H}), 3.54\left(\mathrm{dd},{ }^{3} J=17.0,{ }^{4} J=0.7 \mathrm{~Hz}, 1 \mathrm{H}\right), 1.34(\mathrm{~s}, 9 \mathrm{H}$, $\left.3 \mathrm{CH}_{3}\right) ;{ }^{13} \mathrm{C}$ NMR (100 MHz, DMSO) $\delta 171.06,157.86$, $148.30,147.73,140.27,137.69,136.94,127.92,127.62$, 127.43, 127.07, 126.88, 125.73, 111.55, 61.25, 35.27, 27.79; HR-MS $\left(\right.$ ESI $^{+}$) $\mathrm{m} / z$ Calcd for $\mathrm{C}_{19} \mathrm{H}_{19} \mathrm{BrClN}_{5} \mathrm{O}_{2} \mathrm{~S}$, $[\mathrm{M}+\mathrm{H}]^{+}$496.02041, found 496.02063.

3-Bromo-N'-(4-bromo-5-fluoro-2-nitrobenzoyl)-N'-(tert-butyl)1-(3-chloropyridin-2-yl)-1H-pyrazole-5-carbohydrazide (10k) White solid. M.p: $126-127{ }^{\circ} \mathrm{C}$ yield: $68 \% ;{ }^{1} \mathrm{H}$ NMR $(400 \mathrm{MHz}, \mathrm{DMSO}) \delta 11.05(\mathrm{~s}, 1 \mathrm{H}, \mathrm{N}-\mathrm{H}), 8.62(\mathrm{~d}$, ${ }^{3} J=5.9 \mathrm{~Hz}, 1 \mathrm{H}$, benzene-H), $8.47\left(\mathrm{~d},{ }^{3} J=4.5 \mathrm{~Hz}, 1 \mathrm{H}\right.$, pyridine- $\mathrm{H}), 8.20\left(\mathrm{~d},{ }^{3} J=8.0 \mathrm{~Hz}, 1 \mathrm{H}\right.$, pyridine- $\left.\mathrm{H}\right), 7.70$ $\left(\mathrm{dd},{ }^{3} J=8.1 \mathrm{~Hz},{ }^{4} J=4.7 \mathrm{~Hz}, 1 \mathrm{H}\right.$, pyridine- $\left.\mathrm{H}\right), 7.13(\mathrm{~d}$, ${ }^{3} J=8.0 \mathrm{~Hz}, 1 \mathrm{H}$, benzene- $\left.\mathrm{H}\right), 7.07(\mathrm{~s}, 1 \mathrm{H}$, pyrazole$\mathrm{H}), 1.45\left(\mathrm{~s}, 9 \mathrm{H}, 3 \mathrm{CH}_{3}\right) ;{ }^{19} \mathrm{~F}$ NMR $(471 \mathrm{MHz}$, DMSOD6) $\delta-96.90 ;{ }^{13} \mathrm{C}$ NMR (100 MHz, DMSO) $\delta 166.32$, $162.91,160.36,157.54,148.23,147.67,140.43,140.00$, $136.55,135.52,135.43,130.60,128.27,127.58,127.31$, $115.64,115.38,111.63,109.91,109.68,100.00,61.87$, 27.25; HR-MS $\left(\mathrm{ESI}^{+}\right) \mathrm{m} / z$ Calcd for $\mathrm{C}_{20} \mathrm{H}_{16} \mathrm{Br}_{2} \mathrm{ClFN}_{6} \mathrm{O}_{4}$, $[\mathrm{M}+\mathrm{H}]^{+}$616.93451, found 616.93433; $[\mathrm{M}+\mathrm{Na}]^{+}$ 638.91464, found 638.91453.

$N^{\prime}$-(4-(Benzyloxy)benzoyl)-3-bromo-N'-(tert-butyl)-1-(3-chlor opyridin-2-yl)-1H-pyrazole-5-carbohydrazide (10I)

White solid. M.p: $236-238{ }^{\circ} \mathrm{C}$ yield: $68 \% ;{ }^{1} \mathrm{H}$ NMR (400 MHz, DMSO) $\delta 10.99(\mathrm{~s}, 1 \mathrm{H}, \mathrm{N}-\mathrm{H}), 8.43(\mathrm{dd}$, ${ }^{3} J=4.7 \mathrm{~Hz},{ }^{4} J=1.5 \mathrm{~Hz}, 1 \mathrm{H}$, pyridine-H), 8.15 (dd, ${ }^{3} J=8.1 \mathrm{~Hz},{ }^{4} J=1.5 \mathrm{~Hz}, 1 \mathrm{H}$, pyridine- $\left.\mathrm{H}\right), 7.62(\mathrm{dd}$, ${ }^{3} J=8.1 \mathrm{~Hz},{ }^{4} J=4.7 \mathrm{~Hz}, 1 \mathrm{H}$, pyridine-H), 7.46-7.31 (m, $7 \mathrm{H}$, benzene- $\mathrm{H}), 7.00-6.93(\mathrm{~m}, 2 \mathrm{H}$, benzene- $\mathrm{H}), 6.91$ (s, $1 \mathrm{H}$, pyrazole- $\mathrm{H}), 5.12\left(\mathrm{~s}, 2 \mathrm{H},-\mathrm{CH}_{2}-\right), 1.41(\mathrm{~s}, 9 \mathrm{H}$, $\left.3 \mathrm{CH}_{3}\right) ;{ }^{13} \mathrm{C}$ NMR $(100 \mathrm{MHz}, \mathrm{DMSO}) \delta 171.50,159.95$, $156.79,148.13,147.60,139.93,137.84,137.21,129.49$, $129.44,128.90,128.38,128.23,127.89,127.30,127.27$, 114.21, 110.61, 69.72, 61.11, 27.91; HR-MS $\left(\mathrm{ESI}^{+}\right) \mathrm{m} / \mathrm{z}$ Calcd for $\mathrm{C}_{27} \mathrm{H}_{25} \mathrm{BrClN}_{5} \mathrm{O}_{3}$, $[\mathrm{M}+\mathrm{H}]^{+}$582.09021, found 582.09052 . 
3-Bromo-N'-(tert-butyl)- $N$ '-(4-chloro-3-fluorobenzoyl)-1-(3-c hloropyridin-2-yl)-1H-pyrazole-5-carbohydrazide (10m) White solid. M.p: $269-270{ }^{\circ} \mathrm{C}$; yield: $72 \%$; ${ }^{1} \mathrm{H}$ NMR (400 MHz, DMSO) $\delta 11.12(\mathrm{~s}, 1 \mathrm{H}, \mathrm{N}-\mathrm{H}), 8.44(\mathrm{dd}$, ${ }^{3} J=4.7 \mathrm{~Hz},{ }^{4} J=1.5 \mathrm{~Hz}, 1 \mathrm{H}$, pyridine-H), 8.16 (dd, ${ }^{3} J=8.1 \mathrm{~Hz},{ }^{4} J=1.5 \mathrm{~Hz}, 1 \mathrm{H}$, pyridine-H), $7.64(\mathrm{dd}$, ${ }^{3} J=8.1 \mathrm{~Hz},{ }^{4} J=4.7 \mathrm{~Hz}, 1 \mathrm{H}$, pyridine-H), 7.57 (dd, ${ }^{3} J=7.2 \mathrm{~Hz},{ }^{4} J=1.9 \mathrm{~Hz}, 1 \mathrm{H}$, benzene-H), 7.49-7.33 $(\mathrm{m}, 2 \mathrm{H}$, benzene- $\mathrm{H}), 6.98(\mathrm{~s}, 1 \mathrm{H}$, pyrazole- $\mathrm{H}), 1.42$ $\left(\mathrm{s}, 9 \mathrm{H}, 3 \mathrm{CH}_{3}\right) ;{ }^{19} \mathrm{~F}$ NMR (471 MHz, DMSO-D6) $\delta$ $-113.90 ;{ }^{13} \mathrm{C}$ NMR (100 MHz, DMSO) $\delta 169.60,158.27$ $\left(\mathrm{d}, J_{C-F}=249.5 \mathrm{~Hz}\right), 157.03,156.69,148.04,147.62$, 139.92, 137.36, 134.80, 134.76, 129.79, 128.49, 128.41, 127.99, 127.39, $119.40\left(\mathrm{~d}, J_{\mathrm{C}-\mathrm{F}}=18.1 \mathrm{~Hz}\right), 119.31$, $116.94\left(\mathrm{~d}, J_{\mathrm{C}-\mathrm{F}}=21.4 \mathrm{~Hz}\right), 116.83,110.81,61.55,40.60$, 40.39, 40.19, 39.98, 39.77, 39.56, 39.35, 27.72; HR-MS $\left(\mathrm{ESI}^{+}\right) \mathrm{m} / z$ Calcd for $\mathrm{C}_{20} \mathrm{H}_{17} \mathrm{BrCl}_{2} \mathrm{FN}_{5} \mathrm{O}_{2},[\mathrm{M}+\mathrm{H}]^{+}$ 527.9995, found 528.0013; $[\mathrm{M}+\mathrm{H}]^{+} 549.98189$, found 549.98161 .

N'-(3-Bromo-1-(3-chloropyridin-2-yl)-1H-pyrazole-5-carbonyl)-N-(tert-butyl)-1-methyl-1H-pyrazole-3-carbohydrazide (10n)

White solid. M.p: $234-235{ }^{\circ} \mathrm{C}$ yield: $74 \%$; ${ }^{1} \mathrm{H}$ NMR (400 MHz, DMSO) $\delta 11.17(\mathrm{~s}, 1 \mathrm{H}, \mathrm{N}-\mathrm{H}), 8.46$ (dd, ${ }^{3} J=4.7 \mathrm{~Hz},{ }^{4} J=1.5 \mathrm{~Hz}, 1 \mathrm{H}$, pyridine-H), 8.19 (dd, ${ }^{3} J=8.1 \mathrm{~Hz},{ }^{4} J=1.5 \mathrm{~Hz}, 1 \mathrm{H}$, pyridine-H), 7.64 (dd, ${ }^{3} J=8.1 \mathrm{~Hz},{ }^{4} J=4.7 \mathrm{~Hz}, 1 \mathrm{H}$, pyridine-H), $7.37(\mathrm{~d}$, ${ }^{3} J=2.0 \mathrm{~Hz}, 1 \mathrm{H}$, pyrazole-H), 7.07 (s, $1 \mathrm{H}$, pyrazole-H), $6.44\left(\mathrm{~d},{ }^{3} \mathrm{~J}=2.0 \mathrm{~Hz}, 1 \mathrm{H}\right.$, pyrazole-H), $3.69(\mathrm{~s}, 3 \mathrm{H}), 1.42$ (s, 9H, $\left.3 \mathrm{CH}_{3}\right) ;{ }^{13} \mathrm{C}$ NMR (100 MHz, DMSO) $\delta 164.05$, $157.45,148.12$, 147.63, 139.98, 137.51, 137.27, 136.68, 127.92, 127.43, 127.29, 110.96, 106.38, 61.66, 38.07, 27.74; HR-MS $\left(\mathrm{ESI}^{+}\right) \mathrm{m} / z$ Calcd for $\mathrm{C}_{18} \mathrm{H}_{19} \mathrm{BrClN}_{7} \mathrm{O}_{2},[\mathrm{M}+\mathrm{H}]^{+}$ 480.05449 , found 480.05432 .

N'-(3-Bromo-1-(3-chloropyridin-2-yl)-1H-pyrazole-5-carbony l)-N-(tert-butyl) nicotinohydrazide (100)

White solid. M.p: 203-205 ${ }^{\circ} \mathrm{C}$; yield: $81 \%$; ${ }^{1} \mathrm{H}$ NMR (400 MHz, DMSO) $\delta 11.19(\mathrm{~s}, 1 \mathrm{H}, \mathrm{N}-\mathrm{H}), 8.63-8.50$ ( $\mathrm{m}, 2 \mathrm{H}$, pyridine-H), 8.47-8.39 ( $\mathrm{m}, 1 \mathrm{H}$, pyridine- $\mathrm{H})$, 8.21-8.11 (m, $1 \mathrm{H}$, pyridine- $\mathrm{H}), 7.74\left(\mathrm{~d},{ }^{3} \mathrm{~J}=7.9 \mathrm{~Hz}\right.$, $1 \mathrm{H}$, pyridine- $\mathrm{H}), 7.63\left(\mathrm{dd},{ }^{3} J=8.1 \mathrm{~Hz},{ }^{4} J=4.7 \mathrm{~Hz}, 1 \mathrm{H}\right.$, pyridine-H), $7.40\left(\mathrm{dd},{ }^{3} J=7.5 \mathrm{~Hz},{ }^{4} J=5.1 \mathrm{~Hz}, 1 \mathrm{H}\right.$, pyridine-H), $6.92(\mathrm{~s}, 1 \mathrm{H}$, pyrazole- $\mathrm{H}), 1.44\left(\mathrm{~s}, 9 \mathrm{H}, 3 \mathrm{CH}_{3}\right)$; ${ }^{13} \mathrm{C}$ NMR (100 MHz, DMSO) $\delta 170.02,156.86,150.96$, $147.99,147.82,147.65,139.98,137.33,134.79,133.04$, $127.85,127.34,127.30,123.45,110.81,61.56,27.76$; HR-MS $\left(\mathrm{ESI}^{+}\right) \mathrm{m} / z$ Calcd for $\mathrm{C}_{19} \mathrm{H}_{18} \mathrm{BrClN}_{6} \mathrm{O}_{2},[\mathrm{M}+\mathrm{H}]^{+}$ 477.04359, found 477.04385; $[\mathrm{M}+\mathrm{Na}]^{+}$499.02554, found 499.02576 .
3-Bromo-N'-(tert-butyl)-1-(3-chloropyridin-2-yl)-N'-(3-(trifluo romethyl)benzoyl)-1H-pyrazole-5-carbohydrazide (10p) White solid. M.p: 274-276 ${ }^{\circ} \mathrm{C}$; yield: $67 \%$; ${ }^{1} \mathrm{H}$ NMR (400 MHz, DMSO) $\delta 11.15(\mathrm{~s}, 1 \mathrm{H}, \mathrm{N}-\mathrm{H}), 8.43(\mathrm{dd}$, ${ }^{3} J=4.7 \mathrm{~Hz},{ }^{4} J=1.4 \mathrm{~Hz}, 1 \mathrm{H}$, pyridine-H), $8.13(\mathrm{dd}$, ${ }^{3} J=8.1 \mathrm{~Hz},{ }^{4} J=1.4 \mathrm{~Hz}, 1 \mathrm{H}$, pyridine- $\left.\mathrm{H}\right), 7.81-7.72$ $(\mathrm{m}, 2 \mathrm{H}$, benzene- $\mathrm{H}), 7.68-7.56(\mathrm{~m}, 3 \mathrm{H}$, benzene- $\mathrm{H})$, $6.87\left(\mathrm{~s}, 1 \mathrm{H}\right.$, pyrazole-H), $1.44\left(\mathrm{~s}, 9 \mathrm{H}, 3 \mathrm{CH}_{3}\right) ;{ }^{19} \mathrm{~F} \mathrm{NMR}$ (471 MHz, DMSO-D6) $\delta-61.02 ;{ }^{13} \mathrm{C}$ NMR $(100 \mathrm{MHz}$, DMSO) $\delta 170.37,156.69,148.03,147.62,139.88,138.10$, $137.31,131.42,129.57, \delta 128.88\left(\mathrm{q}, J_{C-F}=32.0 \mathrm{~Hz}\right)$, $128.40,127.94,127.34,127.02\left(\mathrm{q}, J_{C-F}=7.6 \mathrm{~Hz}\right), 125.75$, $124.40\left(\mathrm{q}, J_{C-F}=272.5 \mathrm{~Hz}\right), 123.90\left(\mathrm{q}, J_{C-F}=7.6 \mathrm{~Hz}\right)$, 123.04, 110.68, 61.53, 27.73; HR-MS $\left(\mathrm{ESI}^{+}\right) \mathrm{m} / z$ Calcd for $\mathrm{C}_{21} \mathrm{H}_{18} \mathrm{BrClF}_{3} \mathrm{~N}_{5} \mathrm{O}_{2},[\mathrm{M}+\mathrm{H}]^{+}$544.03573, found 544.03551.

N'-(3-Bromo-1-(3-chloropyridin-2-yl)-1H-pyrazole-5-carbony I)-N-(tert-butyl)-2,6-dichloroisonicotinohydrazide (10q) White solid. M.p: $235-236{ }^{\circ} \mathrm{C}$; yield: $65 \%$; ${ }^{1} \mathrm{H}$ NMR (400 MHz, DMSO) $\delta 11.15(\mathrm{~s}, 1 \mathrm{H}, \mathrm{N}-\mathrm{H}), 8.46$ (dd, ${ }^{3} J=4.7 \mathrm{~Hz},{ }^{4} J=1.5 \mathrm{~Hz}, 1 \mathrm{H}$, pyridine-H), $8.18(\mathrm{dd}$, ${ }^{3} J=8.1 \mathrm{~Hz},{ }^{4} J=1.5 \mathrm{~Hz}, 1 \mathrm{H}$, pyridine-H), $7.67(\mathrm{dd}$, ${ }^{3} J=8.1 \mathrm{~Hz},{ }^{4} J=4.7 \mathrm{~Hz}, 1 \mathrm{H}$, pyridine- $\left.\mathrm{H}\right), 7.42(\mathrm{~s}, 2 \mathrm{H}$, pyridine-H), 7.07 (s, $1 \mathrm{H}$, pyrazole- $\mathrm{H}), 1.42\left(\mathrm{~s}, 9 \mathrm{H}, 3 \mathrm{CH}_{3}\right)$. ${ }^{13} \mathrm{C}$ NMR (100 MHz, DMSO) $\delta 167.14,156.91,150.64$, $149.55,148.02,147.74,139.95,136.82,128.10,127.50$, 121.20, 111.26, 62.20, 27.50; HR-MS $\left(\mathrm{ESI}^{+}\right) \mathrm{m} / z$ Calcd for $\mathrm{C}_{19} \mathrm{H}_{16} \mathrm{BrCl}_{3} \mathrm{~N}_{6} \mathrm{O}_{2}, \quad[\mathrm{M}+\mathrm{H}]^{+}$544.96565, found 544.96541.

3-Bromo-N'-(tert-butyl)-1-(3-chloropyridin-2-yl)-N'-(2-(trifluo romethyl)benzoyl)-1H-pyrazole-5-carbohydrazide (10r)

White solid. M.p: $260-262{ }^{\circ} \mathrm{C}$; yield: $74 \%$; ${ }^{1} \mathrm{H}$ NMR (400 MHz, DMSO) $\delta 10.87(\mathrm{~s}, 1 \mathrm{H}, \mathrm{N}-\mathrm{H}), 8.52(\mathrm{~s}, 1 \mathrm{H}$, pyridine-H), $8.23(\mathrm{~s}, 1 \mathrm{H}$, pyridine- $\mathrm{H}), 7.80-7.65(\mathrm{~m}$, $2 \mathrm{H}$, benzene- $\mathrm{H}+$ pyridine- $\mathrm{H}), 7.57\left(\mathrm{~d},{ }^{3} \mathrm{~J}=6.6 \mathrm{~Hz}\right.$, $2 \mathrm{H}$, benzene- $\mathrm{H}), 7.13(\mathrm{~s}, 1 \mathrm{H}$, pyrazole- $\mathrm{H}), 6.66(\mathrm{~s}, 1 \mathrm{H}$, benzene-H), $1.44\left(\mathrm{~s}, 9 \mathrm{H}, 3 \mathrm{CH}_{3}\right) ;{ }^{13} \mathrm{C} \mathrm{NMR} \mathrm{(100} \mathrm{MHz,}$ DMSO) $\delta 170.37,156.69,148.03,147.62,139.88,138.10$, $137.31,131.42,129.57, \delta 128.88\left(\mathrm{q}, J_{C-F}=32.0 \mathrm{~Hz}\right)$, 128.40, 127.94, 127.34, 127.02 (q, $\left.J_{C-F}=7.6 \mathrm{~Hz}\right), 125.75$, $124.40\left(\mathrm{q}, J_{C-F}=272.5 \mathrm{~Hz}\right), 123.90\left(\mathrm{q}, J_{C-F}=7.6 \mathrm{~Hz}\right)$, 123.04, 110.68, 61.53, 27.73; HR-MS $\left(\mathrm{ESI}^{+}\right) \mathrm{m} / z$ Calcd for $\mathrm{C}_{21} \mathrm{H}_{18} \mathrm{BrClF}_{3} \mathrm{~N}_{5} \mathrm{O}_{2},[\mathrm{M}+\mathrm{H}]^{+}$544.03573, found 544.03557.

3-Bromo-N'-(5-bromo-2-fluorobenzoyl)- $N^{\prime}$-(tert-butyl)-1-(3-c hloropyridin-2-yl)-1H-pyrazole-5-carbohydrazide (10s)

White solid. M.p: $223-224{ }^{\circ} \mathrm{C}$ yield: $72 \%$; ${ }^{1} \mathrm{H}$ NMR (400 MHz, DMSO) $\delta 11.14(\mathrm{~s}, 1 \mathrm{H}, \mathrm{N}-\mathrm{H}), 8.47$ (dd, 
${ }^{3} J=4.7 \mathrm{~Hz},{ }^{4} J=1.5 \mathrm{~Hz}, 1 \mathrm{H}$, pyridine-H), 8.19 (dd, ${ }^{3} J=8.1 \mathrm{~Hz},{ }^{4} J=1.5 \mathrm{~Hz}, 1 \mathrm{H}$, pyridine- $\left.\mathrm{H}\right), 7.65(\mathrm{dd}$, ${ }^{3} J=8.1 \mathrm{~Hz},{ }^{4} J=4.7 \mathrm{~Hz}, 1 \mathrm{H}$, pyridine-H), $7.62(\mathrm{dd}$, $\left.{ }^{3} J=9.4 \mathrm{~Hz},{ }^{4} J=1.8 \mathrm{~Hz}, 1 \mathrm{H}, \mathrm{Ar}-\mathrm{H}\right), 7.38\left(\mathrm{dd},{ }^{3} J=8.2 \mathrm{~Hz}\right.$, $\left.{ }^{4} J=1.8 \mathrm{~Hz}, 1 \mathrm{H}, \mathrm{Ar}-\mathrm{H}\right), 7.11\left(\mathrm{t},{ }^{3} J=7.8 \mathrm{~Hz}, 1 \mathrm{H}, \mathrm{Ar}-\mathrm{H}\right)$, $6.92(\mathrm{~s}, 1 \mathrm{H}$, pyrazole- $\mathrm{H}), 1.42\left(\mathrm{~s}, 9 \mathrm{H}, 3 \mathrm{CH}_{3}\right) ;{ }^{13} \mathrm{C}$ NMR $(100 \mathrm{MHz}, \mathrm{DMSO}) \delta 166.85,157.95\left(\mathrm{~d}, J_{\mathrm{C}-\mathrm{F}}=251.7 \mathrm{~Hz}\right)$ 157.14, 148.06, 147.64, 140.01, 137.21, 130.03 127.97, $127.78,127.42,127.31,125.14\left(\mathrm{~d}, J_{C-F}=17.4 \mathrm{~Hz}\right), 123.17$ $\left(\mathrm{d}, J_{C-F}=9.4 \mathrm{~Hz}\right), 119.41\left(\mathrm{~d}, J_{C-F}=25.0 \mathrm{~Hz}\right) 111.01,61.80$, 27.69; HR-MS $\left(\mathrm{ESI}^{+}\right) \mathrm{m} / z$ Calcd for $\mathrm{C}_{20} \mathrm{H}_{17} \mathrm{Br}_{2} \mathrm{ClFN}_{5} \mathrm{O}_{2}$, $[\mathrm{M}+\mathrm{H}]^{+}$571.94943, found 571.94928, $[\mathrm{M}+\mathrm{Na}]^{+}$ 593.93138, found 593.93181.

3-Bromo-N'-(tert-butyl)-1-(3-chloropyridin-2-yl)-N'-(furan-3carbonyl)-1H-pyrazole-5-carbohydrazide (10t)

White solid. M.p: $221-223{ }^{\circ} \mathrm{C}$ yield: $73 \% ;{ }^{1} \mathrm{H}$ NMR (400 MHz, DMSO) $\delta 11.21(\mathrm{~s}, 1 \mathrm{H}, \mathrm{N}-\mathrm{H}), 8.45$ (dd, ${ }^{3} J=4.7 \mathrm{~Hz},{ }^{4} J=1.5 \mathrm{~Hz}, 1 \mathrm{H}$, pyridine-H), $8.19(\mathrm{dd}$, ${ }^{3} J=8.1 \mathrm{~Hz},{ }^{4} J=1.5 \mathrm{~Hz}, 1 \mathrm{H}$, pyridine-H), $7.96(\mathrm{dd}$, ${ }^{3} J=1.5 \mathrm{~Hz},{ }^{4} J=0.8 \mathrm{~Hz}, 1 \mathrm{H}$, furan-H), 7.67-7.65 (m, $1 \mathrm{H}$, Furan-H), $7.63\left(\mathrm{dd},{ }^{3} J=8.1 \mathrm{~Hz},{ }^{4} J=4.7 \mathrm{~Hz}, 1 \mathrm{H}\right.$, pyridine- $\mathrm{H}), 7.31(\mathrm{~s}, 1 \mathrm{H}$, pyrazole- $\mathrm{H}), 6.65\left(\mathrm{dd},{ }^{3} J=1.9 \mathrm{~Hz}\right.$, ${ }^{4} J=0.8 \mathrm{~Hz}, 1 \mathrm{H}$, furan-H), $1.39\left(\mathrm{~s}, 9 \mathrm{H}, 3 \mathrm{CH}_{3}\right) \cdot{ }^{13} \mathrm{C}$ NMR (100 MHz, DMSO) $\delta 164.93,157.48,148.39,147.62$, 145.52, 143.52, 139.97, 137.53, 128.06, 127.61, 127.36, 122.44, 110.99, 61.47, 27.92; HR-MS $\left(\mathrm{ESI}^{+}\right) \mathrm{m} / z$ Calcd for $\mathrm{C}_{18} \mathrm{H}_{17} \mathrm{BrClN}_{5} \mathrm{O}_{3},[\mathrm{M}+\mathrm{H}]^{+}$466.02761, found 466.02732, $[\mathrm{M}+\mathrm{Na}]^{+} 488.00955$, found 488.00913 .

N'-(3-bromo-1-(3-chloropyridin-2-yl)-1H-pyrazole-5-carbony I)-N-(tert-butyl)-4-(trifluoromethyl)nicotinohydrazide (10u) White solid. M.p: $187-189{ }^{\circ} \mathrm{C}$; yield: $70 \%$; ${ }^{1} \mathrm{H}$ NMR (400 MHz, DMSO) $\delta 11.07(\mathrm{~s}, 1 \mathrm{H}, \mathrm{N}-\mathrm{H}), 8.84(\mathrm{~d}$, ${ }^{3} J=5.1 \mathrm{~Hz}, 1 \mathrm{H}$, pyridine-H), $8.50(\mathrm{~s}, 1 \mathrm{H}$, pyridine- $\mathrm{H})$, $8.21\left(\mathrm{~d},{ }^{3} \mathrm{~J}=7.7 \mathrm{~Hz}, 1 \mathrm{H}\right.$, pyridine-H), $7.80\left(\mathrm{~d},{ }^{3} \mathrm{~J}=5.1 \mathrm{~Hz}\right.$, $1 \mathrm{H}$, pyridine- $\mathrm{H}), 7.67\left(\mathrm{dd},{ }^{3} \mathrm{~J}=7.9 \mathrm{~Hz},{ }^{3} \mathrm{~J}=4.7 \mathrm{~Hz}, 1 \mathrm{H}\right.$, pyridine-H), $6.84(\mathrm{~s}, 1 \mathrm{H}$, pyrazole- $\mathrm{H}), 1.45\left(\mathrm{~s}, 9 \mathrm{H}, 3 \mathrm{CH}_{3}\right)$; ${ }^{19} \mathrm{~F}$ NMR (471 MHz, DMSO-D6) $\delta-60.17 ;{ }^{13} \mathrm{C}$ NMR (100 MHz, DMSO) $\delta 170.83,167.31,151.50,147.93$, $147.76,140.13,137.06,129.88,127.87,127.38,127.28$, $120.75,111.24,62.12,27.34$; HR-MS $\left(\mathrm{ESI}^{+}\right) \mathrm{m} / z$ Calcd for $\mathrm{C}_{20} \mathrm{H}_{17} \mathrm{BrClF}_{3} \mathrm{~N}_{6} \mathrm{O}_{2},[\mathrm{M}+\mathrm{H}]^{+}$545.03098, found 545.03062 .

3-Bromo-N'-(tert-butyl)-1-(3-chloropyridin-2-yl)-N'-(3,4-dichl orobenzoyl)-1H-pyrazole-5-carbohydrazide (10v)

White solid. M.p: $228-225{ }^{\circ} \mathrm{C}$; yield: $71 \%$; ${ }^{1} \mathrm{H}$ NMR (400 MHz, DMSO) $\delta 11.08(\mathrm{~s}, 1 \mathrm{H}, \mathrm{N}-\mathrm{H}), 8.36(\mathrm{dd}, J=4.7$, $1.5 \mathrm{~Hz}, 1 \mathrm{H}$, pyridine- $\mathrm{H}), 8.08\left(\mathrm{dd},{ }^{3} J=8.1 \mathrm{~Hz},{ }^{4} J=1.5 \mathrm{~Hz}\right.$, $1 \mathrm{H}$, pyridine- $\mathrm{H}), 7.58\left(\mathrm{dd},{ }^{3} J=3.4 \mathrm{~Hz},{ }^{4} J=1.3 \mathrm{~Hz}, 1 \mathrm{H}\right.$, Ar-H), $7.56\left(\mathrm{dd},{ }^{3} J=3.2 \mathrm{~Hz},{ }^{4} J=1.4 \mathrm{~Hz}, 1 \mathrm{H}, \mathrm{Ar}-\mathrm{H}\right)$, $7.51\left(\mathrm{~d},{ }^{4} J=2.0 \mathrm{~Hz}, 1 \mathrm{H}, \mathrm{Ar}-\mathrm{H}\right), 7.29\left(\mathrm{~d},{ }^{4} J=1.1 \mathrm{~Hz}, 1 \mathrm{H}\right.$,
Ar-H), $7.26\left(\mathrm{dd},{ }^{3} J=8.3,{ }^{4} J=2.0 \mathrm{~Hz}, 1 \mathrm{H}, \mathrm{Ar}-\mathrm{H}\right), 6.91(\mathrm{~s}$, $1 \mathrm{H}$, pyrazole-H), $1.34\left(\mathrm{~s}, 9 \mathrm{H}, 3 \mathrm{CH}_{3}\right) .{ }^{13} \mathrm{C}$ NMR $(100 \mathrm{MHz}$, DMSO) $\delta 169.54,156.69,148.02,147.61,139.92,137.56$, $137.30,132.93,131.05,130.64,129.32,128.13,128.00$, $127.55,127.40,127.12,110.86,61.63,27.69$; HR-MS $\left(\mathrm{ESI}^{+}\right) \mathrm{m} / z$ Calcd for $\mathrm{C}_{20} \mathrm{H}_{17} \mathrm{BrCl}_{3} \mathrm{~N}_{5} \mathrm{O}_{2},[\mathrm{M}+\mathrm{H}]^{+}$ 543.97040, found 543.97081, $[\mathrm{M}+\mathrm{Na}]^{+}$565.95234, found 565.95271 .

$N^{\prime}$-Benzoyl-3-bromo-N'-(tert-butyl)-1-(3-chloropyridin-2-yl)1H-pyrazole-5-carbohydrazide (10w)

White solid. M.p: $269-270{ }^{\circ} \mathrm{C}$; yield: $78 \%$; ${ }^{1} \mathrm{H}$ NMR (400 MHz, DMSO) $\delta 11.00(\mathrm{~s}, 1 \mathrm{H}, \mathrm{N}-\mathrm{H}), 8.45$ (dd, ${ }^{3} J=4.7 \mathrm{~Hz},{ }^{4} J=1.5 \mathrm{~Hz}, 1 \mathrm{H}$, pyridine-H), 8.17 (dd, ${ }^{3} J=8.1 \mathrm{~Hz},{ }^{4} J=1.5 \mathrm{~Hz}, 1 \mathrm{H}$, pyridine-H), $7.63(\mathrm{dd}$, ${ }^{3} J=8.1,{ }^{4} J=4.7 \mathrm{~Hz}, 1 \mathrm{H}$, pyridine-H), 7.42-7.34 (m, $5 \mathrm{H}$, benzene-H), 6.79 (s, $1 \mathrm{H}$, pyrazole-H), $1.43\left(\mathrm{~s}, 9 \mathrm{H}, 3 \mathrm{CH}_{3}\right)$; ${ }^{13} \mathrm{C}$ NMR (100 MHz, DMSO) $\delta 181.36,172.00,156.91$, $148.08,147.62,139.98,137.72,137.38,130.11,128.13$, 127.90, 127.29, 127.21, 127.12, 110.58, 61.17, 27.83; HR-MS $\left(\mathrm{ESI}^{+}\right) \mathrm{m} / z$ Calcd for $\mathrm{C}_{20} \mathrm{H}_{19} \mathrm{BrClN}_{5} \mathrm{O}_{2},[\mathrm{M}+\mathrm{H}]^{+}$ 476.04834, found 476.04871, $[\mathrm{M}+\mathrm{Na}]^{+}$498.03029, found 498.03072.

\section{3-Bromo-N'-(2-bromo-5-chlorobenzoyl)-N'-(tert-butyl)-1-(3-c hloropyridin-2-yl)-1H-pyrazole-5-carbohydrazide (10x)}

White solid. M.p: $208-210{ }^{\circ} \mathrm{C}$; yield: $72 \%$; ${ }^{1} \mathrm{H}$ NMR (400 MHz, DMSO) $\delta 11.03(\mathrm{~s}, 1 \mathrm{H}, \mathrm{N}-\mathrm{H}), 8.52(\mathrm{~d}$, ${ }^{3} J=3.9 \mathrm{~Hz}, 1 \mathrm{H}$, benzene-H), $8.21\left(\mathrm{dd},{ }^{3} J=8.1 \mathrm{~Hz}\right.$, ${ }^{4} J=1.4 \mathrm{~Hz}, 1 \mathrm{H}$, pyridine-H), $7.67\left(\mathrm{dd},{ }^{3} J=8.1 \mathrm{~Hz}\right.$, ${ }^{4} J=4.7 \mathrm{~Hz}, 1 \mathrm{H}$, pyridine-H), $7.56\left(\mathrm{dd},{ }^{3} J=8.6 \mathrm{~Hz}\right.$, ${ }^{4} J=2.4 \mathrm{~Hz}, 1 \mathrm{H}$, pyridine- $\left.\mathrm{H}\right), 7.42\left(\mathrm{~d},{ }^{3} \mathrm{~J}=8.5 \mathrm{~Hz}, 1 \mathrm{H}\right.$, benzene-H), $6.90(\mathrm{~s}, 1 \mathrm{H}$, pyrazole- $\mathrm{H}), 1.45\left(\mathrm{~s}, 9 \mathrm{H}, 3 \mathrm{CH}_{3}\right)$. ${ }^{13} \mathrm{C}$ NMR (100 MHz, DMSO) $\delta 167.44,157.30,148.15$, $147.75,140.01,137.04,133.41,131.50,129.59,128.21$, $127.40,127.22,119.95,111.11,56.51,27.56$; HR-MS $\left(\mathrm{ESI}^{+}\right) \mathrm{m} / z$ Calcd for $\mathrm{C}_{20} \mathrm{H}_{17} \mathrm{Br}_{2} \mathrm{Cl}_{2} \mathrm{~N}_{5} \mathrm{O}_{2}, \quad[\mathrm{M}+\mathrm{H}]^{+}$ 587.91988, found 587.91951.

\section{Biological assay}

All bioassays were conducted on test organisms reared in the lab and repeated at $25 \pm 1{ }^{\circ} \mathrm{C}$ according to statistical requirements. Mortalities were corrected using Abbott's formula [37]. Evaluations were based on a percentage scale $(0=$ no activity and $100=$ complete eradication $)$, at intervals of $5 \%$.

\section{Insecticidal activity against $\mathrm{H}$. armigera}

The insecticidal activities of some of the synthesised compounds and avermectins against Helicoverpa armigera were evaluated by the diet-incorporated method [33]. A quantity of $3 \mathrm{~mL}$ of prepared solutions containing the compounds was added to the forage ( $27 \mathrm{~g}$ ), subsequently 
diluted to different concentrations and then placed in a 24-pore plate. One larva was placed in each of the wells on the plate. Mortalities were determined after 72-96 h.

\section{Insecticidal activity against P. xylostella}

The insecticidal activities of compounds 10a-10y against third instar larvae of $P$. xylostella were evaluated according to a previously reported procedure [33-35]. Fresh cabbage discs (diameter: $2 \mathrm{~cm}$ ) were dipped into the prepared solutions containing compounds $10 \mathrm{a}-10 \mathrm{y}$ for $10 \mathrm{~s}$, air-dried, and then placed in a Petri dish (diameter: $9 \mathrm{~cm}$ ) lined with filter paper. Then, ten third instar larvae of $P$. xylostella were carefully transferred to the Petri dish. Each assay was conducted in triplicate. Mortality was calculated $72 \mathrm{~h}$ after treatment. The control groups were treated with distilled water containing TW-80 (0.1 mL/L). Commercial insecticides (i.e., chlorantraniliprole, chlorpyrifos, and avermectins) were tested and compared under the same conditions.

\section{Conclusions}

Twenty-four novel 3-bromo-1-(3-chloropyridin-2-yl)$1 H$-pyrazole-5-carbohydrazide derivatives (10a-10x) were designed and synthesized based on combinating the sub-structures of chlorantraniliprole and diacylhydrazines. These compounds were characterized and confirmed by ${ }^{1} \mathrm{H}$ NMR, ${ }^{13} \mathrm{C}$ NMR, HR-MS. A preliminary evaluation of the insecticidal activities of the synthesized compounds was conducted. Most compounds exhibited good insecticidal activity against Helicoverpa armigera and P. xylostella. In particular, the $\mathrm{LC}_{50}$ values of compounds $10 \mathbf{e}, \mathbf{1 0 g}, \mathbf{1 0 h}, \mathbf{1 0 j}$ and $10 x$ were 86.98 , $27.49,23.67,69.07$, and $28.90 \mathrm{mg} \mathrm{L}^{-1}$, respectively. Notably, compounds $10 \mathrm{~g}, 10 \mathrm{~h}$, and $10 \mathrm{x}$ showed much higher insecticidal activity than that of tebufenozide $\left(\mathrm{LC}_{50}=37.77 \mathrm{mg} \mathrm{L}^{-1}\right)$. Preliminary SAR analysis indicated that phenyl, 4-fluoro phenyl and four fluorophenyl had positive influence on the insecticidal activity of synthesized compounds, and introduction of a heterocyclic ring (pyridine and pyrazole) could decrease their insecticidal effects. Further structural modification and biological evaluation to explore the full potential of this kind of 3-bromo-1-(3-chloropyridin-2-yl)-1H-pyrazole-5-carbohydrazide derivatives are currently underway.

\section{Additional file}

Additional file 1. All the copies of ${ }^{1} \mathrm{H} N M R,{ }^{19} \mathrm{~F} \mathrm{NMR}$ and ${ }^{13} \mathrm{C}$ NMR for the title compounds were presented in Additional information.

\section{Authors' contributions}

The current study is an outcome of constructive discussion with JW. YYW, FZX, ALD and ZQL carry out their synthesis and characterization experiments; GY, JS and $\mathrm{CHL}$ performed the insecticidal activities; JHX and FHW carried out the ${ }^{1} \mathrm{H}$
NMR, ${ }^{19} \mathrm{~F} \mathrm{NMR},{ }^{13} \mathrm{C}$ NMR spectral analyses; FZX carried out the HR-MS. JW was also involved in the drafting of the manuscript and revising the manuscript. All authors read and approved the final manuscript.

\section{Acknowledgements}

The National Natural Science Foundation of China (Nos. 21562012, 21302025, 21162004), Special Foundation of S\&T for Outstanding Young Talents in Guizhou (No. 2015-15\#), The S\&T Foundation of Guizhou Province (No. J[2014]2056\#) and the Graduate Innovation Foundation of Guizhou University (No. 2017058) are gratefully acknowledged.

\section{Competing interests}

The authors declare that they have no competing interests.

\section{Publisher's Note}

Springer Nature remains neutral with regard to jurisdictional claims in published maps and institutional affiliations.

Received: 2 May 2017 Accepted: 31 May 2017

Published online: 05 June 2017

\section{References}

1. Wing KD (1988) RH 5849 a nonsteroidal ecdysone agonist: effects on a Drosophila cell line. Science 241:467

2. Aller HE, Ramsay JR (1988) RH-5849-a novel insect growth regulator with a new mode of action. In: Brighton crop prot conf-pests dis. pp 511-518

3. Heller JJ, Mattioda H, Klein E, Sagenmueller A (1992) Field evaluation of $\mathrm{RH} 5992$ on lepidopterous pests in Europe. Brighton crop prot conf-pests dis. pp 59-65

4. Yanagi M, Sugizaki H, Toya T, Kato Y, Shirakura H, Watanabe T, Yajima Y, Kodama S, Masui A (1992) Preparation of hydrazine derivatives and their pesticidal activity. Chem Abstr 117:212514

5. Yanagi M, Watanabe T, Masui A, Yokoi S, Tsukamoto Y, Ichinose R (2000) ANS-118: a novel insecticide. In: BCPC conf-pests dis. pp 27-32

6. Xu N, Zhang Y, Zhang X, Ni J, Xiong J, Shen M (2007) Manufacture of JS118 insecticide suspension agent. Chem Abstr 146:332500

7. Zhang X, Li Y, Zhu L, Liu L, Sha X, Xu H, Ma H, Wang F, Ni Y, Guo L (2001) Preparation of diacylhydrazines insecticides and their intermediates. Chem Abstr 137:294865

8. Cui Z, Zhang L, Huang J, Yang X, Ling Y (2010) Synthesis and bioactivity of novel $N, N^{\prime}$-diacylhydrazine derivatives containing furan (III). Chin J Chem 28:1257-1266

9. Hu C, Liu J, Du X (2016) Synthesis and insecticidal activities of N-(tertbutyl)- $N$ '-fluorobenzoyl-substitutedpyridylcarbonyl hydrazide derivatives. Chin J Org Chem 36:1051-1059

10. Huang ZQ, Liu YX, Li YQ, Xiong L, Cui Z, Song H, Liu H, Zhao QQ, Wang QM (2011) Synthesis crystal structures insecticidal activities and structureactivity relationships of novel $N^{\prime}$-tert-butyl- $N^{\prime}$-substituted-benzoyl- $N$ di(octa)hydro benzofuran\{(2,3-dihydro)benzo 1,3(1,4)dioxine\} carbohydrazide derivatives. J Agric Food Chem 59:635-644

11. Liu C, Zhang J, Zhou Y, Wang B, Xiong L, Li Z (2014) Design synthesis and insecticidal activity of novel anthranilic diamides containing oxime ester and diacylhydrazine moieties. Chem Res Chin Univ 30:228-234

12. Mao CH, Wang KL, Wang ZW, Ou XM, Huang RQ, Bi FC, Wang QM (2008) Synthesis and insecticidal evaluation of novel $N^{\prime}$-tert-butyl- $N^{\prime}$ substitutedbenzoyl-N-5-chloro-6-chromanecarbohydrazide derivatives. Bioorg Med Chem 16:488-494

13. Shang J, Sun RF, Li YQ, Huang RQ, Bi F, Wang QM (2010) Synthesis and insecticidal evaluation of $\mathrm{N}$-tert-butyl- $\mathrm{N}^{\prime}$-thio 1-(6-chloro3-pyridylmethyl)-2-nitroiminoimidazolidine- $N$, $N^{\prime}$-diacylhydrazines. J Agric Food Chem 58:1834-1837

14. Shang J, Wang QM, Huang RQ, Mao CH, Chen L, Bi FC, Song HB (2005) Synthesis crystal structure and biological activity of aryl $\left(N, N^{\prime}\right.$-diacyl- $N^{\prime}$ tert-butylhydrazino)thio methylcarbamates. Pest Manag Sci 61:997-1002

15. Song BA, Luo LJ, Xue W, Wu J, Hu DY, Yang S, Jin LH, Yuan QK, Lv MM (2014) Pyridinyl-pyrazole heterocyclic diacylhydrazine derivative preparation method and application as pesticide. Chem Abstr 160:95044 
16. Sun GX, Sun ZH, Yang MY, Liu XH, Ma Y, Wei YY (2013) Design synthesis biological activities and 3D-QSAR of new $N, N^{\prime}$-diacylhydrazines containing 2,4-dichlorophenoxy moieties. Molecules 18:14876-14891

17. Wang H, Yang Z, Fan Z, Wu Q, Zhang Y, Mi N, Wang S, Zhang Z, Song $H$, Liu F (2011) Synthesis and insecticidal activity of $N$-tert-butyl- $N$, $N^{\prime}$-diacylhydrazines containing 1,2,3-thiadiazoles. J Agric Food Chem 59:628-634

18. Wang QM, Cheng J, Huang RQ (2002) Synthesis and insecticidal evaluation of novel $\mathrm{N}$-(S-amino)sulfenylated derivatives of diacylhydrazines. Pest Manag Sci 58:1250-1253

19. Zhao PL, Li J, Yang GF (2007) Synthesis and insecticidal activity of chromanone and chromone analogues of diacylhydrazines. Bioorg Med Chem 15:1888-1895

20. Zhao QQ, Shang J, Liu YX, Wang K, Bi FC, Huang RQ, Wang QQ (2007) Synthesis and insecticidal activities of novel $N$-sulfenyl- $N^{\prime}$-tert-butyl- $N$, $N^{\prime}$-diacylhydrazines. 1. N-alkoxysulfenate derivatives. J Agric Food Chem 55:9614-9619

21. Sawada Y, Yanai T, Nakagawa H, Tsukamoto Y, Tamagawa Y, Yokoi S, Yanagi M, Toya T, Sugizaki H, Kato Y, Shirakura H, Watanabe T, Yajima Y, Kodama S, Masui A (2003) Synthesis and insecticidal activity of benzoheterocyclic analogues of $N^{\prime}$-benzoyl- $N$-(tert-butyl)benzohydrazide. Part 3. Modification of N-tert-butylhydrazine moiety. Pest Manag Sci 59:49-57

22. Sawada Y, Yanai T, Nakagawa H, Tsukamoto Y, Yokoi S, Yanagi M, Toya T, Sugizaki H, Kato Y, Shirakura H, Watanabe T, Yajima Y, Kodama S, Masui A (2003) Synthesis and insecticidal activity of benzoheterocyclic analogues of $N^{\prime}$-benzoyl-N-(tert-butyl)benzohydrazide: part 2. Introduction of substituents on the benzene rings of the benzoheterocycle moiety. Pest Manag Sci 59:36-48

23. Sawada Y, Yanai T, Nakagawa H, Tsukamoto Y, Yokoi S, Yanagi M, Toya T, Sugizaki H, Kato Y, Shirakura H, Watanabe T, Yajima Y, Kodama S, Masui A (2003) Synthesis and insecticidal activity of benzoheterocyclic analogues of $N^{\prime}$-benzoyl- $N$-(tert-butyl)benzohydrazide. Part 1. Design of benzoheterocyclic analogues. Pest Manag Sci 59:25-35

24. Lahm GP, Stevenson TM, Selby TP, Freudenberger JH, Cordova D, Flexner L, Bellin CA, Dubas CM, Smith BK, Hughes KA, Hollingshaus JG, Clark CE, Benner EA (2007) Rynaxypyr: a new insecticidal anthranilic diamide that acts as a potent and selective ryanodine receptor activator. Bioorg Med Chem Lett 17:6274-6279

25. Hughes KA, Lahm GP, Selby TP, Stevenson TM (2004) Preparation of cyano anthranilamide insecticides. Chem Abstr 141:190786

26. Li K, Chang X, Song Y, Li B, Liu J (2011) Research of biological activity of SYP-9080. Agrochemicals 50:761-763 (in chinese)
27. Wang BL, Zhu HW, Ma Y, Xiong LX, Li YQ, Zhao Y, Zhang JF, Li ZM (2014) Studies on the amide bridge modification of anthranilic diamide insecticides and biological activities based on the insect RyR. In: 248th ACS national meeting \& exposition, San Francisco, CA, United States, August $10-14$

28. Xu J, Dong WL, Xiong LX, Li Y, Li ZM (2009) Design synthesis and biological activities of novel amides (sulfonamides) containing $N$-pyridylpyrazole. Chin J Chem 27:2007-2012

29. Zhao Y, Li YQ, Xiong LX, Xu LP, Peng LN, Li F, Li ZM (2013) Design syntheses and biological activities of novel anthranilic diamide insecticides containing N-pyridylpyrazole. Chem Res Chin Univ 29:51-56

30. Zhou Y, Wang B, Di F, Xiong L, Yang N, Li Y, Li Y, Li Z (2014) Synthesis and biological activities of 2,3-dihydro-1,3,4-oxadiazole compounds and its derivatives as potential activator of ryanodine receptors. Bioorg Med Chem Lett 24:2295-2299

31. Li Z, Zhou Y, Liu C, Zhou S, Di F, Xiong L, Wang B, Li Y, Zhao Y (2014) Preparation of pyrazole derivatives as agricultural insecticides. Chem Abstr 160:190120

32. Wu J, Huang CQ, Wang J, Hu DY, Jin LH, Yang S, Song BA (2013) Separation interconversion and insecticidal activity of the cis- and trans-isomers of novel hydrazone derivatives. J Sep Sci 36:602-608

33. Wu J, Song BA, Hu DY, Yue M, Yang S (2012) Design synthesis and insecticidal activities of novel pyrazole amides containing hydrazone substructures. Pest Manag Sci 68:801-810

34. Wu J, Xie DD, Shan WL, Zhao YH, Zhang W, Song BA, Yang S, Ma J (2015) Synthesis and insecticidal activity of anthranilic diamides with hydrazone substructure. Chem Pap 69:993-1003

35. Kang SH, Song BA, Wu J, He M, Hu DY, Jin LH, Zeng S, Xue W, Yang S (2013) Design synthesis and insecticidal activities of novel acetamido derivatives containing $N$-pyridylpyrazole carboxamides. Eur J Med Chem 67:14-18

36. Wang H, Fu YF, Fan ZJ, Song HB, Wu QJ, Zhang YJ, Belskaya NP, Bakulev VA (2011) Synthesis crystal structure and biological activity of $N$-tertbutyl-N-(4-methyl-1,2,3-thiadiazole)-5-yl- $N^{\prime}$-(4-methyl-1,2,3-thiadiazole)5-formyl- $N^{\prime}-3,5$-dichloropyridin-2-yl-diacylhydrazine. Chin J Struct Chem 30:412-416

37. Abbott WS (1987) A method of computing the effectiveness of an insecticide. 1925. J Am Mosq Control Assoc 3:302-303

\section{Submit your manuscript to a SpringerOpen ${ }^{\circ}$ journal and benefit from:}

- Convenient online submission

Rigorous peer review

- Immediate publication on acceptance

- Open access: articles freely available online

- High visibility within the field

- Retaining the copyright to your article

Submit your next manuscript at $>$ springeropen.com 